\title{
Six-dimensional muon beam cooling using a homogeneous absorber: Concepts, beam dynamics, cooling decrements, and equilibrium emittances in a helical dipole channel
}

\author{
Yaroslav Derbenev \\ Thomas Jefferson National Accelerator Facility, Newport News, Virginia 23606, USA \\ Rolland P. Johnson* \\ Muons, Inc., Batavia, Illinois 60510, USA
}

(Received 15 December 2003; revised manuscript received 15 September 2004; published 29 April 2005)

\begin{abstract}
The fast reduction of the six-dimensional phase space of muon beams is an essential requirement for muon colliders and also of great importance for neutrino factories based on accelerated muon beams. Ionization cooling, where all momentum components are degraded by an energy absorbing material and only the longitudinal momentum is restored by rf cavities, provides a means to quickly reduce transverse beam sizes. However, the beam energy spread cannot be reduced by this method unless the longitudinal emittance can be transformed or exchanged into the transverse emittance. Emittance exchange plans until now have been accomplished by using magnets to disperse the beam along the face of a wedge-shaped absorber such that higher momentum particles pass through thicker parts of the absorber and thus suffer larger ionization energy loss. In the scheme advocated in this paper, a special magnetic channel designed such that higher momentum corresponds to a longer path length, and therefore larger ionization energy loss, provides the desired emittance exchange in a homogeneous absorber without special edge shaping. Normal-conducting rf cavities imbedded in the magnetic field regenerate the energy lost in the absorber. One very attractive example of a cooling channel based on this principle uses a series of high-gradient rf cavities filled with dense hydrogen gas, where the cavities are in a magnetic channel composed of a solenoidal field with superimposed helical transverse dipole and quadrupole fields. In this scheme, the energy loss, the rf energy regeneration, the emittance exchange, and the transverse cooling happen simultaneously. The theory of this helical channel is described in some detail to support the analytical prediction of almost a factor of $10^{6}$ reduction in six-dimensional phase space volume in a channel about $56 \mathrm{~m}$ long. Equations describing the particle beam dynamics are derived and beam stability conditions are explored. Equations describing six-dimensional cooling in this channel are also derived, including explicit expressions for cooling decrements and equilibrium emittances.
\end{abstract}

DOI: 10.1103/PhysRevSTAB.8.041002

PACS numbers: 29.27.-a, 29.20.-c, 14.60.Ef, 41.85.Lc

\section{INTRODUCTION}

The fast reduction of the six-dimensional (6D) phase space of muon beams is an essential requirement for muon colliders [1-3] and also of great importance for neutrino factories [4-6] based on accelerated muon beams. Ionization cooling $[7,8]$ provides a means to quickly reduce transverse beam sizes, but the beam momentum spread cannot be reduced by this method unless the longitudinal emittance can be transformed or exchanged into the transverse emittance.

In the scheme advocated in this paper, a muon beam cooling channel is made of a series of rf cavities filled with high-density hydrogen gas, which provides simultaneous emittance exchange and transverse ionization cooling by virtue of a superimposed helical magnetic field. Coils placed outside of the rf cavities create a solenoidal magnetic field component, which does not change direction, and transverse dipole and quadrupole helical components, which change direction along the channel axis in the same

\footnotetext{
*Corresponding author.

Email address: rol@muonsinc.com
}

manner as is found in spin-rotating magnets used in Siberian snakes [9]. The energy loss, the rf energy regeneration, the emittance exchange, and the transverse cooling happen simultaneously. Except for the pressure windows at the two ends of the channel, the muons pass only through hydrogen for the most efficient cooling possible. As the beam travels down the channel the beam bunches become shorter and smaller such that higher-frequency rf cavities with smaller transverse dimensions can be used to allow more efficient rf parameters and smaller diameter magnets to enable higher fields and gradients.

\section{A. Emittance exchange in a homogeneous absorber}

The idea that is the basis of this paper for a $6 \mathrm{D}$ cooling channel is seen in a comparison of Figs. 1(a) and 1(b). Figure 1(a) is a conceptual picture of the usual mechanism for reducing the energy spread in a muon beam. The dispersion of the beam generated by the dipole magnet in Fig. 1(a) creates an energy-position correlation at a wedgeshaped absorber. Higher energy particles pass through thicker parts of the absorber and so have more energy loss than particles of less energy. After the absorber the beam becomes more monoenergetic. This process is emit- 


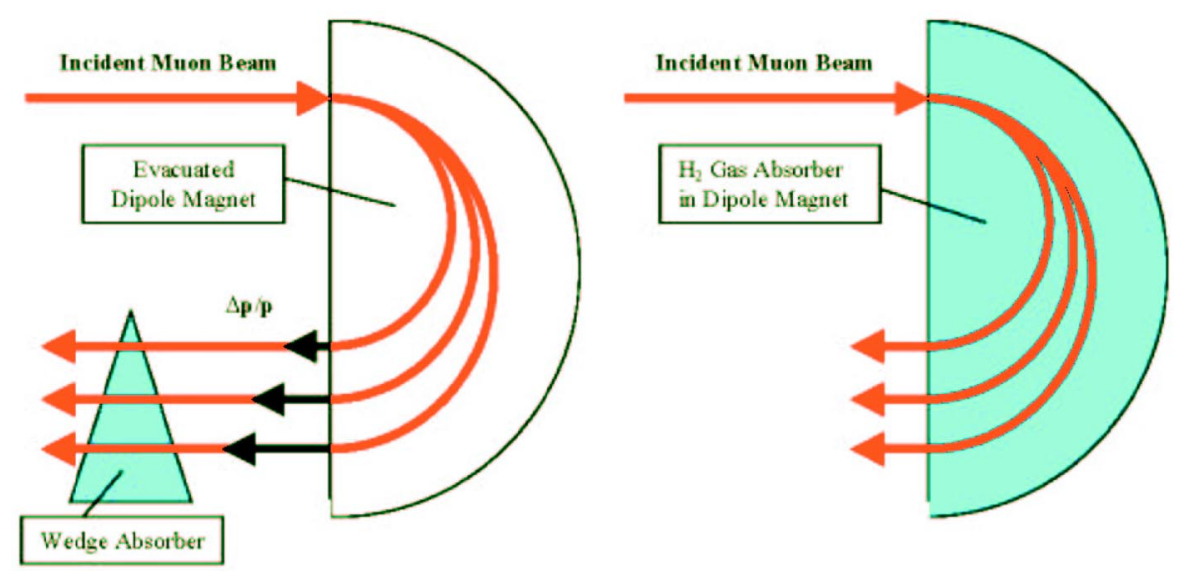

FIG. 1. (Color) (a) Wedge absorber technique and (b) new homogeneous absorber technique.

tance exchange, as it is sometimes called, because the transverse emittance must grow to allow the longitudinal emittance to be cooled. In Fig. 1(a), the beam is in vacuum except in the wedge absorber. Subsequent rf cavities, also in vacuum, replace the energy lost in the absorber. The process is limited by multiple scattering in the absorber and the high- $Z$ windows that isolate the evacuated rf cavities and that contain the absorbers.

In previous cooling plans, both the emittance exchange process and the transverse ionization beam cooling processes have been implemented by sequentially alternating absorbers and evacuated rf cavities. Moreover, the usual $6 \mathrm{D}$ schemes require sequential use of wedge absorbers for emittance exchange followed by unshaped absorbers for transverse cooling followed by rf cavities to regenerate the lost energy.

The new idea advocated in this paper is shown conceptually in Fig. 1(b). In this simpler picture, the coolingchannel magnets are filled with dense gaseous energy absorber. The magnetic dispersion creates a longer path length for particles of higher momentum. The longer path length, in turn, times the absorber $d E / d s$ gives the energy loss correlation with momentum needed for 6D cooling. Thus a homogeneous absorber, without shaped edges, can be used to accomplish emittance exchange. Note that the $180^{\circ}$ geometry shown in the figures is chosen purely to illustrate the principles involved; the geometry of the cooling channel proposed below is quite different.

A second new idea advocated here is that the rf cavities can be inside the cooling-channel magnets and operate while filled with the gaseous energy absorber. Thus the ionization energy loss and the rf energy regeneration can be simultaneous rather than sequential.

\section{B. rf cavities filled with absorber}

The initial concept that a homogeneous absorber, one without shaped edges, would be attractive for emittance exchange is related to the development of rf cavities filled with dense gas [10]. A project [11] presently underway at Fermilab has demonstrated that an $800 \mathrm{MHz}$ rf cell filled with cold, pressurized hydrogen gas can achieve $80 \mathrm{MV} / \mathrm{m}$ with exceptionally short conditioning times [12]. This project is to study the use of high-pressure gases in rf cavities to facilitate large gradients by suppressing high-voltage breakdown by virtue of Paschen's law [13]. A series of contiguous pillbox cavities similar to the one being developed in this project could define the helical cooling channel described in this paper.

Most rf cavities associated with particle accelerators operate in as close to a vacuum as possible to avoid electrical breakdown. This is done so that electrons or ions that are accelerated by the high voltages in the rf cavity rarely encounter atoms of the residual gas, and so the avalanche process of breakdown is inhibited. Other $\mathrm{rf}$ systems that do not require the ultrahigh vacuum of an accelerator typically suppress $\mathrm{rf}$ breakdown by using dense materials between electrodes. Ions passing through these materials, which include high-pressure and/or high-density gases, have such a short mean free path between collisions that they do not accelerate to energies high enough to create an avalanche. The relationship between the electrical breakdown voltage and the product of gas pressure and gap width is known as Paschen's law.

The gas in the cavities also acts as the energy absorber needed for ionization cooling, where hydrogen or helium are the only realistic choices because of their favorable energy loss and radiation length. All things considered, however, hydrogen is superior in all aspects except for perceived safety concerns. Hydrogen gas has over twice the ionization cooling effectiveness as helium in that it allows a final cooled emittance, proportional to $(Z+1)$, that is smaller by a factor of 1.5 in each transverse plane. At the same pressure, hydrogen suppresses rf breakdown at a voltage that is 6 times higher than helium. Hydrogen is also superior in heat capacity and viscosity, which are important parameters for the engineering of a practical cooling channel. 


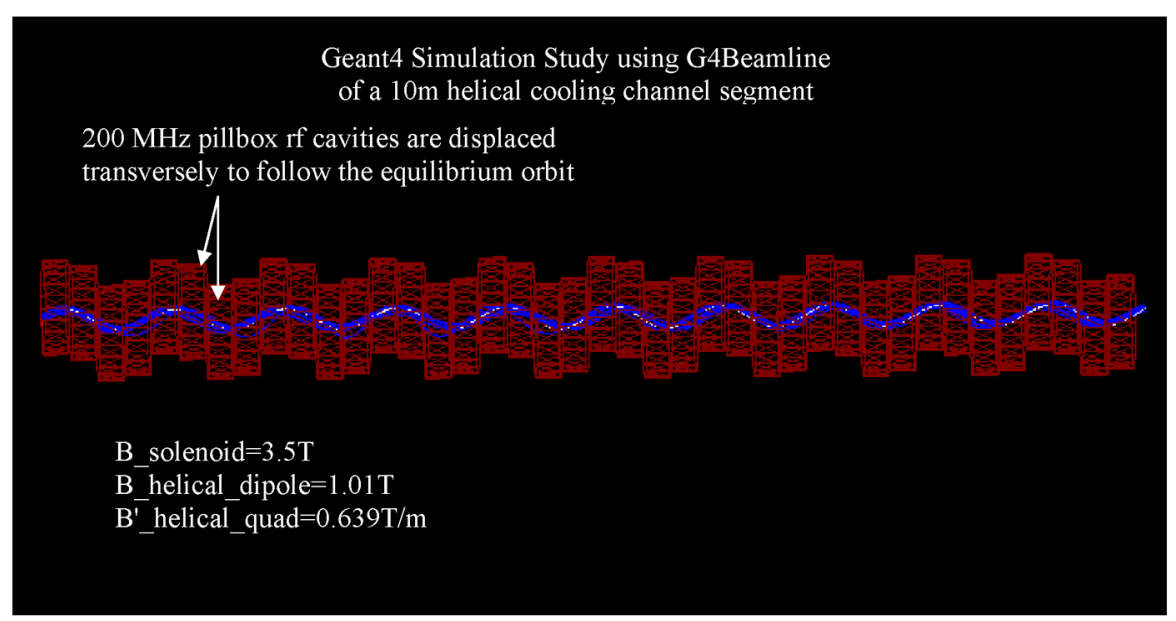

FIG. 2. (Color) G4BEAMLINE display of a 10-m-long segment of a helical cooling channel that is being simulated. The outlines of the forty $200 \mathrm{MHz}$ pillbox rf cavities are shown in red. In this simulation, superimposed magnetic fields with solenoidal and helical components provide focusing and dispersion as the muons pass through the hydrogen-filled rf cavities. Muon trajectories are shown (blue) as they oscillate about the equilibrium orbit (white).

This idea of filling rf cavities with gas is new for particle accelerators and is only possible for muons because they do not scatter as do strongly interacting protons or shower electromagnetically as do less-massive electrons. Additionally, use of a gaseous absorber presents other practical advantages that make it a simpler and more effective cooling method compared to liquid hydrogen flasks in the conventional designs of transverse cooling channels, such as the scheme envisioned by the MICE Collaboration [14] for a demonstration experiment proposed for Rutherford Appleton Laboratory (RAL).

\section{Helical cooling channel segment example}

Figure 2 is a display from G4BEAMLINE [15], a simulation program based on GEANT4 [16], of an initial 10-m-long cooling channel segment, which shows one possible arrangement of pressurized rf cavities. In this example, the rf cavities are displaced transversely from the structure axis such that the muon beam passes through their centers. The $40 \mathrm{rf}$ cavities shown in red in this example are $200 \mathrm{MHz}$ pillbox cavities with $30 \mathrm{~cm}$ diameter aperture. Figure 3 is an end view of the same channel, where the (red) outlines of the rf cavities can be seen. Fifty muon trajectories (blue) are seen relative to the (white) equilibrium orbit.

Superconducting coils (not shown) surround the rf cavities to provide a magnetic field with solenoidal, helical dipole, and helical quadrupole components to create the muon orbits indicated by the blue traces in the figure. The object of this paper is to describe the characteristics of this magnetic channel, which has unusually good acceptance and cooling qualities. The simulations of this channel are underway and will be reported in later papers. Later in this paper, a cooling channel example is described which has three segments such as the one shown here, each with rf frequencies and dimensions adjusted as the beam shrinks to attain optimum cooling rates.

\section{Comparison with ring coolers}

There have been several proposed emittance exchange schemes based on the use of wedge absorbers in muon beam accelerators and storage rings $[1,2,17]$. The most recent progress in the study of $6 \mathrm{D}$ cooling has been with ring coolers (RC), where muons pass several times through a small storage ring with dispersion regions for emittance

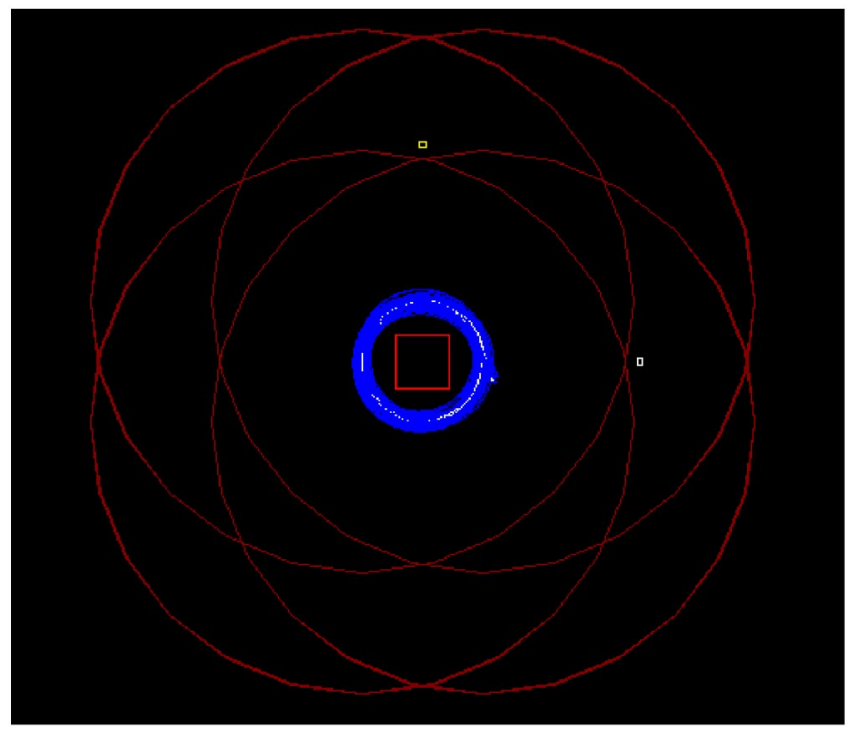

FIG. 3. (Color) The same conditions as Fig. 2, but viewed looking down the cooling channel. The beam here is at $200 \mathrm{MeV} / c$ with a helix radius of $11 \mathrm{~cm}$. The outlines of the radially displaced rf cavities are shown in red. The red box shown for orientation in the center is $10 \mathrm{~cm}$ on a side. 
exchange cooling [18]. RCs are sophisticated devices, where many difficult problems have been addressed using combinations of dipole, solenoidal, and quadrupole magnets interspersed with rf cavities and energy absorbers of different materials and shapes.

Although $6 \mathrm{D}$ cooling is very much required to satisfy the requirements of a muon collider, in the most recent exercises it is looked at more often as a possible way to economize the construction costs of a neutrino factory. In this context, $6 \mathrm{D}$ cooling reduces transverse beam sizes and bunch lengths so that higher frequency and therefore more economical rf can accelerate muons to the energy of the storage ring of a neutrino factory. This acceleration to the storage ring energy in Fermilab and Brookhaven neutrino factory design studies used recirculating linacs, which amounted to more than a quarter of the neutrino factory construction costs.

An RC is in itself a way to economize in that the 15 or so turns the beam makes during the cooling process allows equipment to be reused. A ring is also a rather familiar device for accelerator physicists, where tricks for dispersion creation and simultaneous matching of transverse and longitudinal constraints are known.

All RCs share common difficulties. Injection (and extraction to a lesser extent) is particularly troublesome because it requires a kicker magnet with parameters unlike any that have been built up to now because the initial beam size is large. The space in the ring lattice taken up by the injection/extraction system usually causes some problem because it changes the symmetry of the ring and displaces rf and absorbers, reducing cooling efficiency. The RC must be designed to accommodate the initial conditions of the muon beam when it is injected. As the beam gets cooled, all six dimensions shrink and the RC is less and less a good match to the beam size, especially from the standpoint of dispersion, absorber parameters, and rf frequency. Additional difficulties with ring coolers arise from the multiple passes of the beam through rf cavities and absorbers, where rf beam loading and absorber heating issues are just being addressed.

Since the cooling channel that we are advocating in this paper is essentially a linac filled with absorber, these difficulties are not issues. Injection and extraction, matching to the beam dimensions as the beam cools, and $\mathrm{rf}$ beam loading and absorber heating from multiple passes are not problems for a linear cooling channel.

RCs cannot easily take advantage of the pressurized high-gradient rf cavities being developed by Muons, Inc. and IIT at Fermilab. To use these rf cavities effectively, the entire ring would have to be filled with dense hydrogen gas since pressure windows would be counterproductive. Beam passing through areas without rf cavities would then suffer a large energy loss. Reducing the gas density could reduce the energy loss, but that would diminish the hoped-for gains of the pressurized cavities. Nevertheless, we note that the idea of using gas-filled ring coolers is being investigated with encouraging results [19].

RCs and the helical channel proposed here are similar in two respects. First, neither will easily accept a beam from a pion decay channel without some transverse precooling, $\mathrm{rf}$ bunching, and carefully matched injection parameters. Second, the helical channel has features of a weak focusing storage ring [20] (installed in a vertical plane, with an effective field index calculated below), where the orbits follow a spiral rather than a circular path.

\section{GENERAL TECHNICAL APPROACH}

\section{A. Helical magnets}

The technology of helical dipole magnets [21] is well known, for example, at Brookhaven where helical "Siberian snakes" [22] are used for spin control in RHIC. A helical dipole can be imagined as a superconducting dipole magnet wound on a beam tube, much like a Tevatron superconducting dipole, twisted about its axis so that the dipole bending field rotates its direction as the particle passes down the tube. A schematic of a helical dipole magnet [23] is shown in Fig. 4. We note that for initial ionization cooling of a muon beam, the helical magnets may require 4 times the aperture than has been used up to now $(\sim 30 \mathrm{~cm})$. Also note that the field of a helical dipole magnet is not intuitively obvious. For example, depending on the dimensions and period, the longitudinal and the transverse components can be comparable and have significant gradients. Given the right initial conditions, a particle will pass through this magnet on a helical trajectory about the magnet axis.

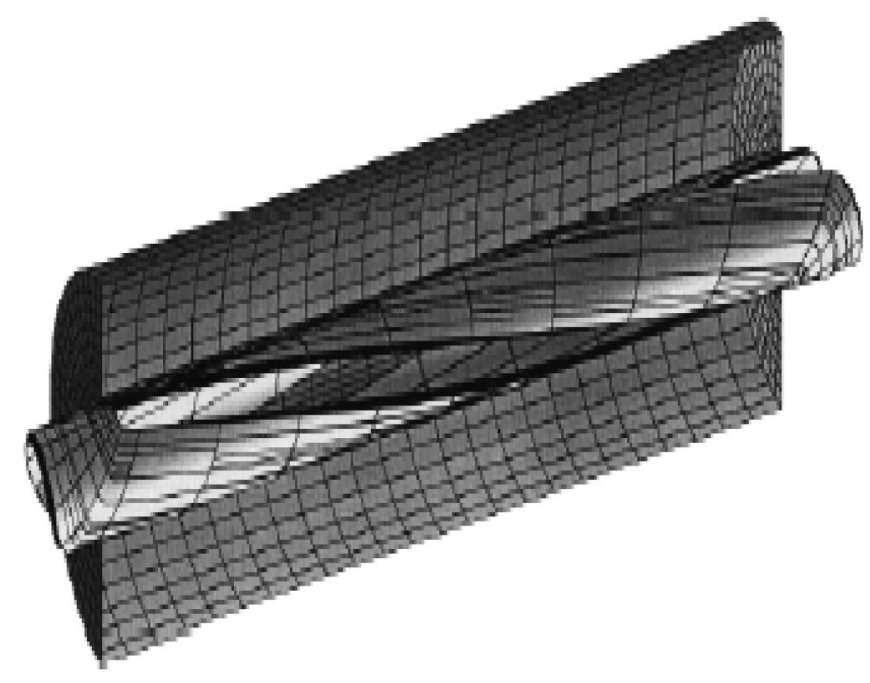

FIG. 4. Schematic representation of a helical dipole magnet showing the coil configuration and a cutaway view of the iron flux-return cylinder. 


\section{B. Helical cooling channel}

The path of a muon traveling down a solenoid without helical magnets is also a helix, where the muon momentum transverse to the axis of the solenoid generates a projected circular orbit with the usual Larmor or cyclotron radius and frequency. If the muons pass through an energy absorber as is required for ionization cooling, the momentum and the cyclotron radius are reduced. This damping of transverse momentum in a solenoid without helical magnets is the basis for most schemes to accomplish transverse ionization cooling. Namely, rf cavities replace the energy lost in the absorber, boosting the beam momentum in the longitudinal direction so that the angular spread $\left(p_{\perp} / p_{z}\right)$ is reduced until it is limited by multiple scattering in the absorber [24].

However, in order to cool the 6D emittance of a beam, the longitudinal motion must be moved to the transverse directions where ionization cooling is effective, as indicated in Fig. 1(b). This emittance exchange is accomplished in the channel proposed in this paper by superimposing a transverse helical dipole magnet to the solenoid on the same axis to make possible longitudinal as well as transverse cooling. As will be shown, the helical dipole magnet creates an outward radial force due to the longitudinal momentum of the particle while the solenoidal magnet creates an inward radial force due to the transverse momentum of the particle, or

$$
\begin{aligned}
F_{h \text {-dipole }} & \approx p_{z} \times B_{\perp} ; \quad b \equiv B_{\perp}, \\
F_{\text {sol }} & \approx-p_{\perp} \times B_{z} ; \quad B \equiv B_{z},
\end{aligned}
$$

where $B$ is the field of the solenoid, the axis of which defines the $z$ axis, and $b$ is the field of the transverse helical dipole at the particle position. These are the Lorentz forces that are the starting point (3.6) for the derivations of the stability conditions for particle motion in these fields in Sec. III. By moving to the rotating or helical frame of reference that follows the field of the helical dipole magnet, a time and $z$-independent Hamiltonian is then developed to explore the characteristics of particle motion in the magnetic fields of the channel. After this, a continuous homogeneous energy absorber is added along with the "continuous" rf cavities needed to compensate for the energy loss and thus maintain the radius of the equilibrium orbit. Equations describing six-dimensional cooling in this channel are also derived, including explicit expressions for cooling decrements and equilibrium emittances.

Some of the actual theoretical development of this cooling channel was worked out some years ago by one of the authors [17]. In that work, the absorber was seen as composed of a homogeneous part and a part with a density gradient. Since the thinking at the time was that the wedge absorber scheme shown in Fig. 1(a) should be dominant, especially in that discrete absorbers were always envisioned, the contributions from the homogeneous absorber were not considered as significant. The ideas and mathematical descriptions become more transparent in the case of a continuous homogeneous absorber. Much of the conceptual simplicity is lost in the case of discrete absorbers that must be carefully placed between magnetic coils and between $\mathrm{rf}$ cavities.

For a given beam momentum, one can vary the solenoid field and the strength and period of the helical dipole field. (The hydrogen gas energy-absorber density is also a free parameter provided the density is sufficient to suppress $\mathrm{rf}$ breakdown at the required level.) As we will demonstrate below, the helical field that must be superimposed on the solenoidal field must have a quadrupole component in addition to the dipole component in order to give the beam additional stability. This component could be added with " $\cos 2 \theta$ " quadrupole magnets having the same twist period as, and superimposed on, the helical dipole coils.

It is important to note that the direction of the solenoidal field does not change in the cooling channel described below. This is an essential difference between the helical dipole method and the solenoidal schemes with alternating field directions that have been envisioned up to now. This may also be some technical advantage to the extent that the large magnetic forces on the superconducting coils at the field reversal regions can be eliminated. Although a discussion of technical issues should follow the complete analysis of beam dynamics and cooling, we note that the use of continuous (or long) solenoids inherent in the helical concept should allow a higher maximum effective longitudinal field than that of schemes with alternating solenoidal field directions. Consequently, the helical scheme will achieve a smaller equilibrium emittance, faster cooling rate, and decreased particle loss from decay.

\section{HELICAL ORBIT DYNAMICS}

Beam dynamics in a helical channel has been studied for free electron lasers using a specific structure with only odd transverse field harmonics and a solenoid [25] and for a structure including a quadrupole harmonic but no solenoid [26]. Below we reproduce an analysis performed earlier [17] for the general case. In developing the cooling theory for a helical beam channel, the only important requirement is that the beam size $\sigma_{\perp}$ should be small with respect to the helix parameter, $\lambda / 2 \pi$. A small beam momentum spread is not assumed.

\section{A. Notation}

In this paper it is assumed that

$-e=1$, in order to simplify later equations involving magnetic fields;

$c=1$

$\vec{p}$ is the particle vector momentum;

$E=\gamma m=\sqrt{p^{2}+m^{2}} ; \beta=\sqrt{1-\left(1 / \gamma^{2}\right)} ;$

$k=2 \pi / \lambda$, where $\lambda$ is the transverse field period, and also the helical orbit period; 
$a$ is the helix orbit radius (a function of $p$ );

$\kappa=k a$ is $a$ in terms of $\lambda / 2 \pi$, such that $\kappa=p_{\perp} / p_{z}$ for the periodic orbit;

$D=p \frac{d a}{d p}$ is the dispersion; $\hat{D}=D / a$ is the dispersion factor;

$x, y, z$ is the Cartesian laboratory frame;

$\vec{\rho}=(x, y)$ is the particle transverse coordinate relative to the structure axis;

$\rho$ and $\varphi$ are the axial coordinates: $x+i y=\rho e^{i \varphi}$;

$\hat{x}$ is the complex transverse coordinate in the rotating or helical frame:

$$
\hat{x}=(x+i y) e^{-i k z}=\rho \exp [i(\varphi-k z)] ;
$$

$\vec{B}=(0,0, B)$ is the solenoid field;

$$
\begin{aligned}
& k_{c}=B \sqrt{1+\kappa^{2}} / p ; \\
& q=\left(k_{c} / k\right)-1 ;
\end{aligned}
$$

$b$ is the transverse magnetic field value at the periodic orbit;

$\vec{b}(x, y, z)$ is the 3 -vector of the periodic field:

$$
\vec{b}(x, y, z+\lambda)=\vec{b}(x, y, z) .
$$

The relationship between the helical magnetic field in the two frames is

$$
b_{x}+i b_{y}=\left(b_{\rho}+i b_{\varphi}\right) e^{i \varphi},
$$

where $b_{\rho}, b_{\varphi}$, and $b_{z}$ are functions of only $\rho$ and $\psi \equiv$ $\varphi-k z$;

$Q_{+}$and $Q_{-}$are $Q$ values or tunes, i.e., transverse oscillation frequencies about the periodic orbit;

$\psi_{+}$and $\psi_{-}$are the phases of free transverse oscillations $\left(\psi_{ \pm}^{\prime}=k Q_{ \pm}\right)$

$\bar{I}_{+}$and $I_{-}$are the corresponding action variables (adiabatic invariants, or generalized Courant-Snyder invariants);

$\Lambda_{+}$and $\Lambda_{-}$are the transverse cooling decrements $\left(I_{ \pm}^{\prime}=-\Lambda_{ \pm} I_{ \pm}\right.$after averaging over $\left.\psi_{ \pm}\right)$;

$\Lambda_{\gamma}$ is the energy cooling decrement:

$$
\Lambda_{\gamma}=-\frac{d}{d \gamma}\left\langle\gamma^{\prime}\right\rangle
$$

where the brackets \langle\rangle mean averaging over free transverse oscillations (i.e., $\psi_{ \pm}$) and $z$.

Figure 5 shows some of the important geometrical relationships between the magnetic fields and particle coordinates used below. Consider a particle of momentum $p$ at a radius of $a(p)$ in a stable helical orbit about the $z$ axis of the structure, which is parallel to the solenoidal field $B$. The parameter $\kappa=p_{\perp} / p_{z}$ is the tangent of the pitch angle of the helical orbits. In the numerical example in Sec VI, this angle is $45^{\circ}$ and $\kappa=1$ as shown in the figure. The transverse helical dipole field $b$ is orthogonal to the $z$ axis and to the radius vector $a$. The beam has to be specially prepared and injected into the channel so that the net transverse

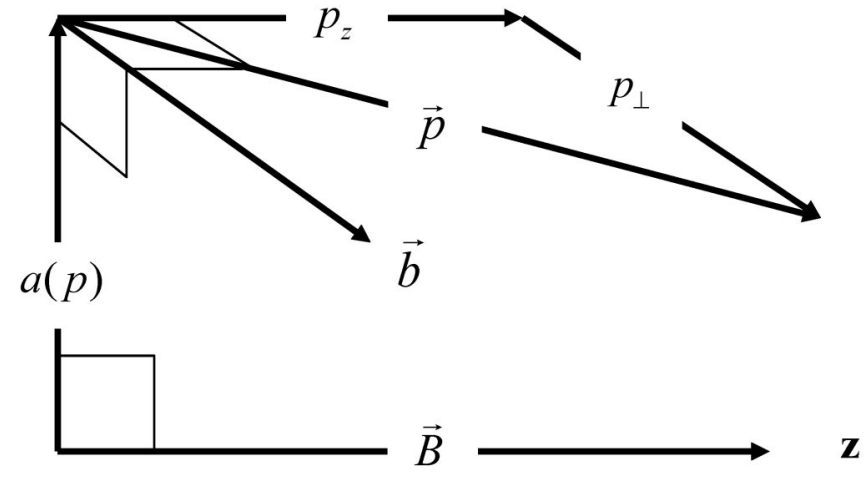

FIG. 5. Diagram of important relationships. The $z$ axis coincides with the solenoid axis. At the periodic orbit, the transverse helical dipole field $b$ is orthogonal to $B$ and the radius vector $a$. The pitch angle of the helix is the $\arctan \left(\kappa=p_{\perp} / p_{z}\right)$.

displacements and divergences of its centroid are matched to the helical equilibrium orbit.

Figure 6 is an illustration of how the beam moves around the structure axis under the influence of the transverse dipole and solenoidal fields. The periodic equilibrium orbit is a helix of constant radius; particles oscillate transversely about this orbit and oscillate longitudinally with respect to the rf with frequencies or tunes as described below. The motion is in the gaseous hydrogen energy absorber that fills the volume of a contiguous series of pillbox rf cavities, which continuously replenish the lost energy. Superconducting coils outside the rf cavities provide the magnetic field of the helical channel.

\section{B. Helical field}

A static magnetic field in vacuum can be represented as a gradient vector of a scalar function:

$$
\vec{b}=\vec{\nabla} U
$$

where $U(\vec{r})$ satisfies the equation $\nabla^{2} U=0$.

In the case of a helical structure, $U$ should reflect helical invariance by being a function of $\psi \equiv \varphi-k z$ and $\rho$ :

$$
U(\vec{r})=U(\rho, \psi)
$$

Then

$$
b_{\rho}=\frac{\partial U}{\partial \rho}, \quad b_{\varphi}=\frac{\partial U}{\rho \partial \varphi}, \quad b_{z}=-k \rho b_{\varphi} .
$$

The Fourier expansion

$$
U=\sum_{\ell \neq 0} U_{\ell}(\rho) e^{i \ell \psi}
$$

leads to an equation for $U_{\ell}(\rho)$ :

$$
\frac{1}{\rho} \frac{d}{d \rho}\left(\rho \frac{d U_{\ell}}{d \rho}\right)-\left(\frac{1}{\rho^{2}}+k^{2}\right) \ell^{2} U_{\ell}=0 .
$$

A solution regular at $\rho=0$ is a modified Bessel function: 


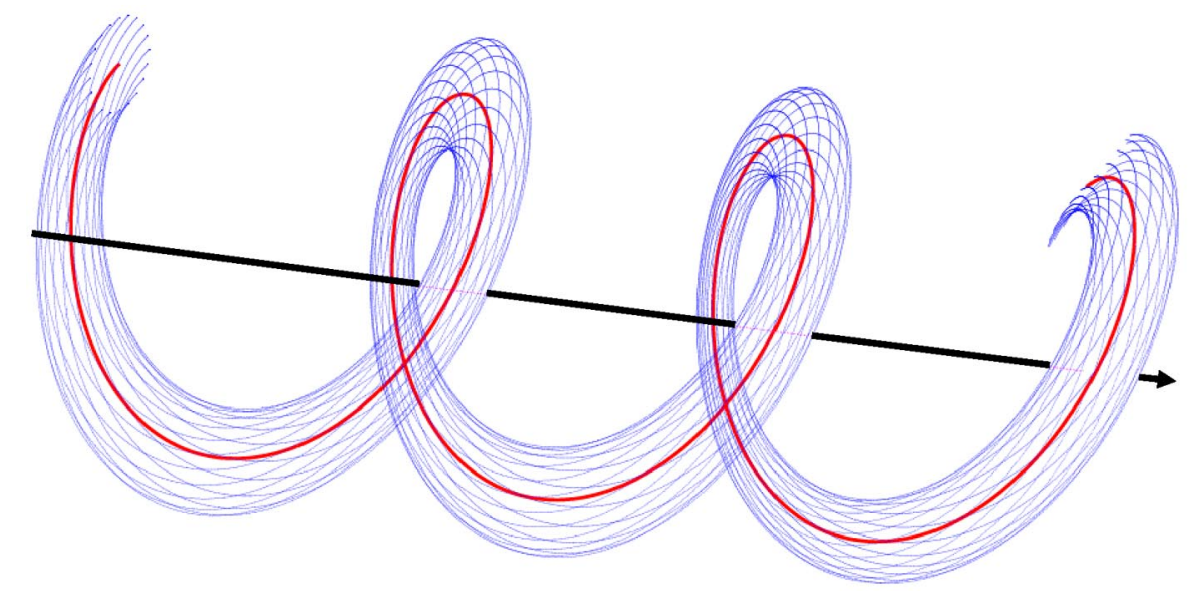

FIG. 6. (Color) Illustration of motion of the beam about the $z$ axis (black), which coincides with the solenoid center. For a given momentum, muons (blue) oscillate about the periodic equilibrium orbit (red). This view in perspective shows 20 muons as they oscillate about the equilibrium orbit for three helix periods.

$$
\begin{gathered}
U_{\ell}=\text { const } \times I_{\ell}(\ell k \rho), \\
I_{\ell}(t)=\left(\frac{t}{2}\right)^{\ell} \sum_{n=0}^{\infty} \frac{\left(t^{2} / 4\right)^{n}}{n !(n+\ell) !} \rightarrow \begin{cases}\left(\frac{t}{2}\right)^{\ell} / \ell ! & t \ll 1 \\
\frac{e^{t}}{\sqrt{2 \pi t}} & t \gg \ell^{2} .\end{cases}
\end{gathered}
$$

Each harmonic is independent and corresponds to a current distribution

$$
j_{z}, \quad j_{\varphi} \propto e^{i \ell \psi}
$$

The most important harmonics are the dipole $(\ell=1)$,

$$
\begin{gathered}
b_{\varphi}=2 b_{d} I_{1}(k \rho)(\cos \psi) / k \rho, \quad b_{\rho}=2 b_{d} I_{1}^{\prime}(k \rho) \sin \psi, \\
b_{z}=-k \rho b_{\varphi},
\end{gathered}
$$

and quadrupole $(\ell=2)$ :

$$
\begin{aligned}
b_{\varphi} & =\left(\frac{\partial b_{\varphi}}{\partial \rho}\right)_{o} \frac{I_{2}(2 k \rho)}{k^{2} \rho} \cos 2\left(\psi-\psi_{2}\right), \\
b_{\rho} & =\frac{1}{k}\left(\frac{\partial b_{\varphi}}{\partial \rho}\right)_{o} I_{2}^{\prime}(2 k \rho) \sin 2\left(\psi-\psi_{2}\right), \\
b_{z} & =-k \rho b_{\varphi}, \quad \psi_{2}=\mathrm{const}
\end{aligned}
$$

where $b_{d}$ and $\left(\frac{\partial b_{\varphi}}{\partial \rho}\right)_{o}$ are the dipole and quadrupole strengths at $\rho=0$, respectively. Sextupole $(\ell=3)$ and octupole $(\ell=4)$ harmonics might be needed for particular improvements. We note that this description corresponds to a field produced by simple external conductors and that other helical configurations of conductors are possible that would best be described by complementing the field description above with MacDonald functions. In fact, our considerations below rely only on the helical invariance of the fields and not on the details of the magnet design.

\section{Equations of motion in a helical field}

Here we derive the equations of motion in the absence of absorbers and rf fields. Since the corresponding forces for absorbers and rf are weak, these effects can be treated using perturbative methods after the magnetic dynamical problem is solved. The Cartesian coordinates are the two transverse coordinates $x, y$, and the longitudinal coordinate $z$ which coincides with the axis of the magnetic structure, having unit vectors $\vec{e}_{x}, \vec{e}_{y}, \vec{e}_{z}$. In the following calculations we use the definitions and relationships from above and

$$
\frac{d z}{d t} \equiv(\dot{z}), \quad \beta_{z} d t=d z, \quad \frac{d}{d z} \equiv\left({ }^{\prime}\right), \quad \vec{\rho}=(x, y),
$$

$$
\vec{p}_{\perp}=p_{z} \vec{\rho}^{\prime}, \quad \frac{p_{z}}{p}=\frac{\beta_{z}}{\beta}=\frac{1}{\sqrt{1+\left(\vec{\rho}^{\prime}\right)^{2}}} .
$$

Let us use the initial Lorentz equation

$$
\dot{\vec{\rho}}=\left(B \vec{e}_{z}+\vec{b}\right) \times \vec{\beta}
$$

in order to separate out the equation for transverse motion

$$
\dot{\vec{\rho}}_{\perp}=\left(B+b_{z}\right) \vec{e}_{z} \times \vec{\beta}_{\perp}+\beta_{z} \vec{b}_{\perp} \times \vec{e}_{z} .
$$

Using (3.4) and (3.5), and complex representation with

$$
u=x+i y, \quad i^{2}=-1,
$$

we can rewrite (3.7) as follows:

$$
\left(p_{z} u^{\prime}\right)^{\prime}=i\left(B+b_{z}\right) u^{\prime}-i\left(b_{x}+i b_{y}\right) .
$$

This equation is complemented by the expression for $p_{z}^{\prime}$, which can easily be obtained using the conservation of total momentum $p$,

$$
p_{z}^{\prime}=-\frac{1}{2} p \frac{\left(\left|u^{\prime}\right|^{2}\right)^{\prime}}{\left(1+\left|u^{\prime}\right|^{2}\right)^{3 / 2}} .
$$


Next, transform Eq. (3.8) to the rotating (helical) frame $x_{1}, x_{2}, z$ with unit vectors $\vec{e}_{1}(z), \vec{e}_{2}(z), \vec{e}_{z}$, where the subscript 1 corresponds to the radial coordinate and field directions and the subscript 2 indicates the azimuthal coordinate and field directions.

Using the relationships

$$
\begin{gathered}
\hat{x} \equiv x_{1}+i x_{2}=u e^{-i k z}, \\
u^{\prime}=\left(\hat{x}^{\prime}+i k \hat{x}\right) e^{i k z}, \\
\vec{\rho}=x \vec{e}_{x}+y \vec{e}_{y}=x_{1} \vec{e}_{1}+x_{2} \vec{e}_{2}, \\
\vec{e}_{x}+i \vec{e}_{y}=\left(\vec{e}_{1}+i \vec{e}_{2}\right) e^{i k z},
\end{gathered}
$$

and

$$
b_{x}+i b_{y}=\left(b_{1}+i b_{2}\right) e^{i k z} \text {, }
$$

we find

$$
\begin{aligned}
p_{z}\left(\hat{x}^{\prime \prime}+2 i k \hat{x}^{\prime}-k^{2} \hat{x}\right)+\left(p_{z}^{\prime}-i B-i b_{z}\right) & \left(\hat{x}^{\prime}+i k \hat{x}\right) \\
& +i\left(b_{1}+i b_{2}\right)=0,
\end{aligned}
$$

with

$$
\begin{aligned}
& p_{z}=\frac{p}{\sqrt{1+\left|\hat{x}^{\prime}+i k \hat{x}\right|^{2}}}, \\
& p_{z}^{\prime}=-\frac{1}{2} p \frac{\left(\left|\hat{x}^{\prime}+i k \hat{x}\right|^{2}\right)^{\prime}}{\left(1+\left|\hat{x}^{\prime}+i k \hat{x}\right|^{2}\right)^{3 / 2}} .
\end{aligned}
$$

Recall that

$$
b_{z}=k\left(x_{2} b_{1}-x_{1} b_{2}\right),
$$

and the transverse field components $b_{1}$ and $b_{2}$ are functions of only $x_{1}$ and $x_{2}$, but not $z$. Thus, the transverse $x_{1}$ and $x_{2}$ dynamics in the helical frame is conservative, although the equations are coupled and nonlinear.

\section{Helical orbits \\ 1. Periodic orbits}

It follows from the periodicity of the helical fields that there will be periodic orbits with the same periodicity. The periodic orbit is determined as a solution of (3.10) at

$$
\begin{aligned}
\rho=\text { const } \equiv a, & x_{1}=a, \quad x_{2}=0, \\
\varphi^{\prime} & =\text { const }=k, \quad \text { i.e., } \psi^{\prime}=0, \quad \psi=0, \\
b_{2}=b_{2}(k a) \equiv b, & b_{1}(\psi=0)=0, \quad p_{z}=\frac{p}{\sqrt{1+\kappa^{2}}} .
\end{aligned}
$$

The equilibrium equation is obtained as follows:

$$
\frac{k_{c}}{k}-1=\frac{\left(1+\kappa^{2}\right)^{3 / 2}}{k p \kappa} b,
$$

or

$$
p(a)=\frac{\sqrt{1+\kappa^{2}}}{k}\left[B-\frac{1+\kappa^{2}}{\kappa} b\right]
$$

or

$$
\frac{b}{B}=\frac{\kappa}{1+\kappa^{2}}\left(1-\frac{k}{k_{c}}\right)=\frac{\kappa}{1+\kappa^{2}}\left(\frac{q}{q+1}\right) .
$$

\section{Dispersion}

The dispersion factor

$$
\hat{D}=\frac{p}{a} \frac{d a}{d p}
$$

plays a key role in the emittance exchange effect. It can be found immediately using the equilibrium Eq. (3.12):

$$
\frac{p}{a} \frac{d a}{d p}=\left(\frac{a}{p} \frac{d p}{d a}\right)^{-1} ;
$$

the result can be expressed as

$$
\hat{D}^{-1}=\frac{\kappa^{2}+\left(1-\kappa^{2}\right) q}{1+\kappa^{2}}+g,
$$

where the effective field index at the periodic orbit is

$$
g \equiv \frac{-\left(1+\kappa^{2}\right)^{3 / 2}}{p k^{2}} \frac{\partial b}{\partial a} .
$$

Note that the dispersion factor $\hat{D}$ does not vanish at $a \rightarrow 0$, while the dispersion $D=\hat{D} a$ disappears.

\section{E. Transverse oscillations about the periodic orbit}

\section{Tunes of the helical orbits}

Consider a position $u_{1}, u_{2}$, relative to the periodic orbit

$$
u_{1}=x_{1}-a(p) ; \quad u_{2}=x_{2} .
$$

Assume $b_{1}=0$ and $\left(\frac{\partial b_{2}}{\partial \psi}\right)=0$ at $\psi=0$. After a linear expansions of Eq. (3.10) with

$$
k^{2}\left(u_{1}^{2}+u_{2}^{2}\right) \ll 1, \quad\left(u_{1}^{\prime}\right)^{2}+\left(u_{2}^{\prime}\right)^{2} \ll 1,
$$

and taking into account the field laws and equilibrium relationship in (3.11), we obtain two linear equations:

$$
u_{1}^{\prime \prime}+\frac{q-1}{1+\kappa^{2}} k u_{2}^{\prime}+k^{2} \hat{D}^{-1} u_{1}=0
$$

and

$$
u_{2}^{\prime \prime}-(q-1) k u_{1}^{\prime}+k^{2}(q-g) u_{2}=0 .
$$

These equations are conservative but coupled. The coupling is due to a difference between the Coriolis force and the nonequilibrium part of the Lorentz force of the solenoid. At $k_{c}=2 k, u_{1}$ and $u_{2}$ become decoupled (in linear approximation). In the following development we do not assume that $u_{1}$ and $u_{2}$ are decoupled, but treat the general case. The solution of these equations can be found as 
eigenvectors

$$
\left(\begin{array}{l}
u_{1} \\
u_{2}
\end{array}\right)=\left(\begin{array}{l}
c_{1} \\
c_{2}
\end{array}\right) e^{i k Q z}, \quad \text { at }\left(\begin{array}{l}
c_{1} \\
c_{2}
\end{array}\right)=\text { const }
$$

where we obtain the zero determinant equation:

$$
Q^{4}-2 Q^{2} R+G=0
$$

with

$$
G=(q-g) \hat{D}^{-1}=\left(\frac{2 q+\kappa^{2}}{1+\kappa^{2}}-\hat{D}^{-1}\right) \hat{D}^{-1}
$$

and

$$
R \equiv \frac{1}{2}\left(1+\frac{q^{2}}{1+\kappa^{2}}\right)
$$

Thus, the $Q$ values are found:

$$
Q^{2}=Q_{ \pm}^{2} \equiv R \pm \sqrt{R^{2}-G}
$$

and the stability area is established:

$$
0<G<R^{2} \text {. }
$$

Note that

$$
Q_{+}^{2} \cdot Q_{-}^{2}=G
$$

while

$$
Q_{+}^{2}+Q_{-}^{2}=2 R
$$

Thus we have two tunes for the normal mode oscillations. For a solenoidal field alone, there is only a single frequency, but when the helical field is added the frequency is split.

\section{Transverse oscillations} ior:

Using Eq. (3.18), we find the normal oscillation behav-

$$
X_{ \pm} \equiv\left|\begin{array}{c}
u_{1} \\
u_{1}^{\prime} \\
u_{2} \\
u_{2}^{\prime}
\end{array}\right|_{ \pm}=a_{ \pm}\left|\begin{array}{c}
\cos \psi_{ \pm} \\
-k Q_{ \pm} \sin \psi_{ \pm} \\
\left(\alpha_{ \pm} / Q_{ \pm}\right) \sin \psi_{ \pm} \\
k \alpha_{ \pm} \cos \psi_{ \pm}
\end{array}\right|,
$$

where $a_{ \pm}=$const, $\psi_{ \pm}=Q_{ \pm} k z+$ const, and

$$
\alpha_{ \pm}=\frac{1+\kappa^{2}}{q-1}\left(Q_{ \pm}^{2}-\hat{D}^{-1}\right) .
$$

We note the useful relationships

$$
\hat{D} \alpha_{+} \alpha_{-}=-\left(1+\kappa^{2}\right)
$$

and

$$
\frac{Q_{+}^{2}}{\alpha_{+}}-\frac{Q_{-}^{2}}{\alpha_{-}}=\frac{\alpha_{+}-\alpha_{-}}{1+\kappa^{2}}=\frac{2}{q-1} \sqrt{R^{2}-G}
$$

The general solution $X$ is a sum of $X_{ \pm}$and the helix path:

$$
\left|\begin{array}{c}
x_{1} \\
x_{1}^{\prime} \\
x_{2} \\
x_{2}^{\prime}
\end{array}\right|=X_{+}+X_{-}+\left|\begin{array}{c}
a(p) \\
0 \\
0 \\
0
\end{array}\right|
$$

\section{Amplitudes}

The solution in (3.27) can be treated also as a transformation from $x_{1}, x_{1}^{\prime}, x_{2}, x_{2}^{\prime}$ to variables of amplitudes and phases of normal mode oscillations. This is reasonable to the extent that perturbative forces related to nonlinearities or nonadiabatic changes of fields and absorbers are small during a single oscillation period. The squared amplitudes are of primary importance. The transformed relationships can be easily found using (3.27) and (3.30)

$$
x_{2}^{\prime}-k \alpha_{\mp}\left(x_{1}-a\right)=\left(\alpha_{ \pm}-\alpha_{\mp}\right) k a_{ \pm} \cos \psi_{ \pm},
$$

and

$$
\alpha_{\mp} x_{1}^{\prime}+k Q_{\mp}^{2} x_{2}=\frac{1}{Q_{ \pm}}\left(\alpha_{ \pm} Q_{\mp}^{2}-\alpha_{\mp} Q_{ \pm}^{2}\right) k a_{ \pm} \sin \psi_{ \pm} .
$$

Thus,

$$
k^{2} a_{+}^{2}=\frac{\left[x_{2}^{\prime}-\alpha_{-} k\left(x_{1}-a\right)\right]^{2}}{\left(\alpha_{+}-\alpha_{-}\right)^{2}}+Q_{+}^{2} \frac{\left(\alpha_{-} x_{1}^{\prime}+Q_{-}^{2} k x_{2}\right)^{2}}{\left(\alpha_{+} Q_{-}^{2}-\alpha_{-} Q_{+}^{2}\right)^{2}}
$$

and

$$
k^{2} a_{-}^{2}=\frac{\left[x_{2}^{\prime}-\alpha_{+} k\left(x_{1}-a\right)\right]^{2}}{\left(\alpha_{+}-\alpha_{-}\right)^{2}}+Q_{-}^{2} \frac{\left(\alpha_{+} x_{1}^{\prime}+Q_{+}^{2} k x_{2}\right)^{2}}{\left(\alpha_{+} Q_{-}^{2}-\alpha_{-} Q_{+}^{2}\right)^{2}} .
$$

\section{Transverse adiabatic invariants}

The effective beam volume in phase space is measured in terms of action variables, or adiabatic invariants $I_{ \pm}$, which are canonically conjugate with the oscillation phases $\psi_{ \pm}$. The action variables are proportional to squared amplitudes:

$$
I_{ \pm}=\frac{1}{2} \beta \gamma k Q_{ \pm} a_{ \pm}^{2} \cdot R_{ \pm},
$$

where we have introduced the coefficients $R_{ \pm}(E)$. To find them, one can use the canonical relationships (see the Appendix)

$$
\frac{\partial}{\partial E} I_{ \pm}\left(\vec{P}_{\perp}, \vec{\rho}, E\right)=-\frac{\partial}{\partial \psi_{ \pm}} t\left(I_{ \pm}, \psi_{ \pm}\right)
$$

where the energy $(-E)$ and time $(t)$ are considered as one of three "old" canonical pairs, together with $\vec{p}_{\perp}$ and $\vec{\rho}$ (while the $z$ coordinate is treated as the "time argument"). To determine the time $t$ as function of "new" variables 
$\left(a_{ \pm}, \psi_{ \pm}\right)$, one has to integrate the equation

$$
t^{\prime}=\frac{1}{\beta_{z}}
$$

along a "solved" particle trajectory. In linear approximation for free particle oscillations near the helical orbit $\vec{a}(p, z)$, we have (see the Appendix)

$$
\begin{aligned}
t & =\hat{t}+\frac{\kappa}{\beta \sqrt{1+\kappa^{2}}}\left[\frac{1+\alpha_{+}}{Q_{+}} a_{+} \sin \psi_{+}+\frac{1+\alpha_{-}}{Q_{-}} a_{-} \sin \psi_{-}\right], \\
\hat{t}^{\prime} & =\text { const }
\end{aligned}
$$

where we have introduced a "shortened time" $\hat{t}$ as a new canonical variable conjugate to the energy, $-E$. Using the relationships (3.32) and (3.37), the shortened time can be expressed as a function of initial variables:

$$
\hat{t}=t+\frac{1}{\beta \sqrt{1+\kappa^{2}}}\left[D x_{1}^{\prime}+\left(1-\frac{q-1}{1+\kappa^{2}} \hat{D}\right) \kappa x_{2}\right] .
$$

Note that the rate $\hat{t}$ changes is constant on a particle trajectory in a magnetic field.

Returning to the derivatives $\frac{\partial a_{ \pm}^{2}}{\partial E}$, they can be found using the relationships in (3.35)-(3.37), in which the variables of the rotating frame $\left(x_{1}, x_{1}^{\prime}, x_{1}, x_{2}^{\prime}\right)$ are to be considered as functions of $\left(x, p_{x}\right),\left(y, p_{y}\right)$ and energy $E$. The calculation in the Appendix gives

$$
\frac{\partial}{\partial E} a_{ \pm}^{2}=\mp \frac{2 a\left(1+\kappa^{2}\right)}{e \beta^{2}} \frac{1+\alpha_{ \pm}}{\alpha_{ \pm}} \alpha_{ \pm} \cos \psi_{ \pm} .
$$

Using Eqs. (3.35)-(3.37) gives

$$
R_{ \pm}= \pm \frac{\alpha_{ \pm}}{\left(1+\kappa^{2}\right)^{3 / 2}} \frac{\alpha_{+}-\alpha_{-}}{Q_{ \pm}^{2}} .
$$

Thus solutions and relationships (3.31)-(3.40) provide a full set of canonical transformations from the initial variables $(\vec{p}, \vec{r})$ to action and phase variables in a magnetic field before a rf field has been introduced.

\section{Beam envelopes on a helical orbit}

In the rotating frame of the helical orbit, each of the two normal mode oscillations is recognized in transverse space as an elliptical orbit with tune $Q_{+}$or $Q_{-}$[see Eq. (3.27)]:

$$
\begin{aligned}
& \left|\begin{array}{l}
u_{1} \\
u_{2}
\end{array}\right|=a_{+}\left|\begin{array}{c}
\cos \psi_{+} \\
\left(\alpha_{+} / Q_{+}\right) \sin \psi_{+}
\end{array}\right|, \\
& \left|\begin{array}{l}
u_{1} \\
u_{2}
\end{array}\right|_{-}=a_{-}\left|\begin{array}{c}
\cos \psi_{-} \\
\left(\alpha_{-} / Q_{-}\right) \sin \psi_{-}
\end{array}\right| .
\end{aligned}
$$

With random phase distributions, there are two normal beam ellipsoids of aspect ratios

$$
\left(\frac{\sigma_{1}}{\sigma_{2}}\right)_{+}=\frac{Q_{+}}{\alpha_{+}}, \quad\left(\frac{\sigma_{1}}{\sigma_{2}}\right)_{-}=\frac{Q_{-}}{\alpha_{-}} .
$$

For ensembles with adiabatic invariants $I_{ \pm}$, one can average over $\psi_{ \pm}$to find the related sizes $\sigma_{1}$ and $\sigma_{2}$ :

$$
\begin{aligned}
& \left(\sigma_{1}\right)_{ \pm}^{2}= \pm \frac{I_{ \pm} Q_{ \pm}}{\gamma \beta k \alpha_{ \pm}\left(\alpha_{+}-\alpha_{-}\right)}, \\
& \left(\sigma_{2}\right)_{ \pm}^{2}=\left(\sigma_{1}\right)_{ \pm}^{2}\left(\frac{\alpha_{ \pm}}{Q_{ \pm}}\right)^{2} .
\end{aligned}
$$

Thus, $\sigma_{1}$ and $\sigma_{2}$ rms sizes can be explicitly found as determined by the two canonical (and uncorrelated) emittances $\varepsilon_{+}$and $\varepsilon_{-}$by substituting for $I_{ \pm}$with $\left\langle I_{ \pm}\right\rangle=\varepsilon_{ \pm}$ into (3.43) and adding a contribution due to energy spread:

$$
\begin{aligned}
\sigma_{1}^{2} & =D^{2}\left(\frac{\Delta p}{p}\right)^{2}+\left(\sigma_{1}\right)_{+}^{2}+\left(\sigma_{1}\right)_{-}^{2}, \\
\sigma_{2}^{2} & =\left(\sigma_{2}\right)_{+}^{2}+\left(\sigma_{2}\right)_{-}^{2} .
\end{aligned}
$$

The canonical emittances $\varepsilon_{ \pm}$will not be disturbed by an adiabatic change of beam optics parameters along the beam path in the helical transport line. These uncorrelated emittances (i.e., the averaged values of adiabatic or generalized Courant-Snyder invariants) also can be conserved in transfers from the helical channel to sections with different optics, such as a conventional alternating gradient transport with uncoupled planes. However, special optics will be needed to match such a transition in order to avoid an increase of beam emittances.

\section{F. Longitudinal oscillations in a rf field}

A rf field has to be applied to compensate for energy loss in an absorber to achieve substantial ionization cooling. Thus the beam must first be captured and bunched before the cooling described in this paper can be accomplished. The capture and bunching processes will be described in a paper to follow this one. In this section we introduce the basic characteristics of longitudinal dynamics of particles in a bunched beam subject to a rf field on a helical beam path without absorber.

\section{Basic equations}

Longitudinal motion of particles in a rf field is governed by equations for energy $E$ and time $t$ as a pair of canonically conjugate variables, with the $z$ coordinate considered a time argument and the rf wave number $k_{\mathrm{rf}}$ expressed in terms of the rf cavity resonant frequency and particle longitudinal velocity,

$$
k_{\mathrm{rf}} \approx \omega / \beta_{z} .
$$

Assuming, as usual, that the change of particle energy along one period of magnetic and electric field and "betatron" oscillations is small, we can express the equations in terms of energy and shortened or average time $t$, given by Eqs. (3.37) and (3.38). A convenient variable is a reference time

$$
\tau=\hat{t}-\left(k_{\mathrm{rf}} / \omega\right) z
$$


Note that the rate of change of $\hat{t}$ as a function of $z$ is not influenced by particle transverse oscillations in a focusing magnetic field. Neglecting the phase $\omega \tau$ and the rate of change of energy on transverse amplitudes, we obtain the shortened canonical equations

$$
\gamma^{\prime}=-\gamma_{\max }^{\prime} \sin \omega \tau=-\frac{\partial}{\partial \tau} H_{S}
$$

and

$$
\tau^{\prime}=\frac{1}{\beta} \sqrt{1+\kappa^{2}}-\frac{k_{\mathrm{rf}}}{\omega}=\frac{\partial}{\partial \gamma} H_{S}
$$

where

$$
H_{S}=\int\left(\frac{1}{\beta} \sqrt{1+\kappa^{2}}-\frac{k_{\mathrm{rf}}}{\omega}\right) d \gamma-\frac{\gamma_{\mathrm{max}}}{\omega} \cos \omega \tau
$$

is the effective longitudinal or synchrotron Hamiltonian, and $\gamma_{\max }^{\prime}$ is the maximum rate of energy change due to the rf field.

The Hamiltonian and Eqs. (3.46) and (3.47) determine the equilibrium phase $\omega \tau=0$, the equilibrium or resonance energy $\gamma_{\text {res }}$, such that

$$
\left(\frac{1}{\beta} \sqrt{1+\kappa^{2}}\right)_{\gamma=\gamma_{\mathrm{res}}}=\frac{k_{\mathrm{rf}}}{\omega},
$$

and the phase space trajectory of particle oscillations around the equilibrium orbit where

$$
H_{S}(\gamma, \tau)=\text { const. }
$$

\section{Translational mobility of a particle on a helical path}

For a particle of energy $E=m \gamma$, the rate of change of phase is given in linear approximation by

$$
\hat{\tau}^{\prime}=\eta\left(\gamma-\gamma_{\text {res }}\right) \equiv \eta \Delta \gamma,
$$

where we have introduced a new parameter, $\eta$, the translational mobility:

$$
\eta=\frac{d}{d \gamma} \frac{\sqrt{1+\kappa^{2}}}{\beta}=\frac{\sqrt{1+\kappa^{2}}}{\gamma \beta^{3}}\left(\frac{\kappa^{2}}{1+\kappa^{2}} \hat{D}-\frac{1}{\gamma^{2}}\right) .
$$

This parameter is analogous to the momentum slip factor in a synchrotron, where the factor $\frac{\kappa^{2}}{1+\kappa^{2}} \hat{D}$ can be identified with $\frac{1}{\gamma_{T}^{2}}$.

\section{Synchrotron tune}

The rate of change of energy for a particle with phase $\omega \tau$ is given in linear approximation by

$$
\Delta \gamma^{\prime}=-\gamma_{\max }^{\prime} \omega \tau
$$

Equations (3.51) and (3.53) determine the synchrotron tune, the frequency of phase and energy oscillations near the equilibrium where $\tau=0$ and $\gamma=\gamma_{\text {res }}$ :

$$
\tau^{\prime \prime}+\omega^{2} Q_{S}^{2} \tau=0 \quad \text { and } \quad Q_{S}^{2}=\eta \gamma_{\max }^{\prime} / \omega
$$

\section{Synchrotron adiabatic invariant, canonical phase, and admittance}

The adiabatic invariant of oscillations about the equilibrium is determined as an area in phase space bounded by the ellipse given by Eq. (3.50):

$$
I_{S}=\frac{1}{2 \pi} \oint \Delta \gamma d \tau
$$

For a small oscillation near the equilibrium, $I_{S}$ is proportional to the Hamiltonian function:

$$
I_{S} \approx \frac{H_{S}}{\omega Q_{S}}=\frac{\eta}{2 \omega Q_{S}}(\Delta \gamma)^{2}+\frac{\omega Q_{S}}{2 \eta} \tau^{2} .
$$

More generally, there is the relationship

$$
d H_{S}=\omega Q_{S}\left(I_{S}\right) d I_{S},
$$

with $Q_{S}\left(I_{S}\right)$ as the amplitude-dependent synchrotron frequency that takes into account the nonlinear behavior of the rf field as a function of phase $\omega \tau$. A canonical variable conjugate to the synchrotron invariant $I_{S}$ is the synchrotron phase $\Psi_{S}$, which varies uniformly on an unperturbed orbit:

$$
\Psi_{S}^{\prime}=\omega Q_{S}\left(I_{S}\right) .
$$

The maximum $I_{S}$ value for captured particles, the admittance, corresponds to oscillations near the synchrotron separatrix:

$$
H_{S}=\frac{\gamma_{\max }}{\omega} \quad \text { and } \quad\left(I_{S}\right)_{\mathrm{adm}}=\frac{2}{\pi \omega} \sqrt{\frac{\gamma_{\max }^{\prime}}{\eta \omega}} .
$$

On the separatrix trajectory, the frequency $Q_{S}$ is zero because the motion stops at the points $\omega \tau= \pm \pi$.

\section{COOLING DECREMENTS}

\section{A. Absorber drag force}

Muons passing through an absorber experience energy and momentum loss due to collisions with electrons. The collision effect averaged over impact parameters is described by the well-known formula for the drag force:

$$
\dot{\vec{p}}=\frac{-F \vec{p}}{p}, \quad F=\frac{4 \pi Z n e^{4} \log }{m_{e} \beta^{2}},
$$

where $Z$ and $n$ are the absorber atomic number and concentration, $m_{e}$ the electron mass, and $\beta$ the muon velocity. Here log is a symbol for the Coulomb logarithm of ionization energy loss for fast particles:

$$
\log \equiv \ln \left(\frac{2 p^{2}}{h \nu m_{\mu}}\right)-\beta^{2},
$$

with $h \nu$ the effective ionization potential [27]. A typical 
magnitude of the $\log$ is about 12 for the conditions described below.

Similar to the radiation force for relativistic electrons in a magnetic field, the drag force produces a damping effect on muon transverse oscillations in a focusing field:

$$
\vec{p}_{\perp}^{\prime}=-\Lambda_{0} \vec{p}_{\perp}, \quad \Lambda_{0}=\frac{F}{\gamma \beta^{2} m_{\mu}} .
$$

Unlike the radiation force, however, the drag force cannot damp the beam energy spread since its characteristic energy dependence, $d F / d E$, is negative or is too small when it is positive $\left(\gamma^{2}>\log \right)$ :

$$
\begin{aligned}
& E^{\prime}=-F(E), \quad \Delta E^{\prime}=-(d F / d E) \Delta E, \\
& (d F / d E)=2 \Lambda_{0}\left[-\frac{1}{\gamma^{2}}+\frac{1-(\beta / \gamma)^{2}}{\log }\right] .
\end{aligned}
$$

To achieve longitudinal cooling requires emittance exchange with transverse oscillations as discussed in Sec. II. Emittance exchange, in turn, requires the introduction of a beam bend that creates dispersion, a correlation between the orbit and energy of a particle. Use of a continuous homogeneous absorber, rather than wedges at discrete points, implies a positive dispersion along the entire cooling path, a condition that we have shown exists for an appropriately designed helical dipole channel. We have also shown that this condition is compatible with stable periodic orbits.

The linear theory of the damping process including wedge effects was developed earlier [15] and later applied and developed in some detail for a homogenous absorber [17]. The treatment in this paper follows the analysis in these previous works but includes more detail. Below we will calculate the longitudinal and transverse damping rates along a helical transport line in a homogeneous absorber with rf fields. As an example of how the cooling rates can be manipulated, we will then indicate how to achieve balanced 6D cooling, where the three decrements are equal.

\section{B. Longitudinal decrement}

\section{Synchrotron oscillations in the absorber}

Energy loss due to muon collisions with absorber electrons can be included after averaging over collision parameters and transverse oscillations about the energydependent helical orbit:

$$
\gamma=-\gamma_{\max }^{\prime} \sin \omega \tau-\frac{F}{m_{\mu}} \sqrt{1+\kappa^{2}}
$$

Expanding energy loss in a linear approximation as a function of energy near the reference orbit, we obtain synchrotron oscillation equations

$$
\Delta \gamma^{\prime}=-\gamma^{\prime}\left(\sin \omega \tau-\sin \omega \tau_{e q}\right)-\Lambda_{\gamma} \Delta \gamma,
$$

where

$$
\gamma_{\max }^{\prime} \sin \omega \tau_{e q}=-\left.\frac{F}{m_{\mu}} \sqrt{1+\kappa^{2}}\right|_{\gamma=\gamma_{\mathrm{res}}},
$$

and we have introduced the energy decrement as

$$
\begin{aligned}
\Lambda_{\gamma} & =\frac{d}{d \gamma} \frac{\langle F\rangle}{m_{\mu}} \sqrt{1+\kappa^{2}} \\
& =\left[-\frac{2}{\gamma^{2}}+\frac{2\left[1-(\beta / \gamma)^{2}\right]}{\log }+\hat{D} \frac{\kappa^{2}}{1+\kappa^{2}}\right] \Lambda_{0} .
\end{aligned}
$$

For positive $\Lambda_{\gamma}$, synchrotron oscillations will damp with a characteristic exponent $\Lambda_{\gamma}$. Note that the synchrotron oscillations in the absorber may last some time since

$$
\Lambda_{\gamma} \ll \omega Q_{S},
$$

where $Q_{S}^{2}=\eta \omega \gamma_{\max }^{\prime} \cos \omega \tau_{e q}$.

The relationship (4.8) follows from the condition $\gamma^{\prime} \ll$ $\omega \gamma_{\text {res }}$, taking into account that $\Lambda \sim \gamma_{\max }^{\prime} / \gamma_{\text {res }}$ unless the translational mobility parameter $\eta$ is very small. Consequently we can continue to treat the phase $\omega \tau$ and energy motion in terms of oscillations even though the reduction in the synchrotron potential well might not be small $\left(F \leq m \gamma^{\prime}\right)$. We can characterize the oscillations by a modified synchrotron adiabatic invariant as determined by the integral in (3.55), but with a Hamiltonian

$$
\hat{H}=\frac{\eta}{2}(\Delta \gamma)^{2}-\gamma_{\max }^{\prime}\left(\cos \omega \tau+\omega \tau \sin \omega \tau_{e q}\right) / \omega .
$$

Damping due to an emittance exchange mechanism can then be considered a slow process.

\section{Synchrotron oscillation decrement}

Under condition (4.8) one can calculate the damping rate of $I_{S}$ using a perturbative method. Considering the adiabatic invariant a function of $\Delta \gamma$ and $\omega \tau$ we find the instantaneous and average rates of change of the adiabatic invariant:

$$
I_{S}^{\prime}=-\frac{\partial I_{S}}{\partial \gamma} \Lambda_{\gamma} \Delta \gamma
$$

Using the canonical relationship $\frac{\partial I_{S}}{\partial \gamma}=\frac{\partial \tau}{\partial \Psi_{S}}$ and taking into account that

$$
\tau^{\prime}=\frac{\partial \tau}{\partial \Psi_{S}} \Psi_{S}^{\prime}=\frac{\partial \tau}{\partial \Psi_{S}} \omega Q_{s}\left(I_{S}\right)
$$

we find a simple damping equation:

$$
\left\langle I_{S}^{\prime}\right\rangle=-\Lambda_{\gamma} \oint \Delta \gamma d \tau / 2 \pi=-\Lambda_{\gamma} I_{S}
$$

Note that the linear reduction of the Hamiltonian has not been used in this derivation. Thus in the approximation of a constant energy decrement as in (4.5), the nonlinearity of synchrotron oscillations does not affect the cooling rate. 
Note, finally, that the instantaneous rate of change of phase $\omega \tau$ is influenced by the transverse component of the drag force ( $\left.\sim x_{1}^{\prime}\right)$ according to Eq. (3.38), but the average effect is zero:

$$
\left\langle\tau \delta \tau^{\prime}\right\rangle \sim\left\langle\tau \frac{\partial \hat{t}}{\partial x_{1}^{\prime}} x_{1}^{\prime}\right\rangle=0
$$

\section{Transverse decrements}

To derive the transverse rates, we have to calculate the average partial power $\left\langle I_{ \pm}^{\prime}\right\rangle$. This can be easily done considering $I_{ \pm}$as functions of transverse vector momentum $\vec{p}_{\perp}$, coordinate $\vec{\rho}$, and energy $E=m \gamma$ :

$$
I_{ \pm}^{\prime}=\frac{1}{\beta_{z}} \frac{\partial I_{ \pm}}{\partial \vec{p}_{\perp}} \vec{F}_{\perp}+\frac{\partial I_{ \pm}}{\partial \gamma} \cdot \gamma^{\prime}
$$

with

$$
\begin{aligned}
\vec{F}_{\perp} & =-F \cdot \frac{\vec{\beta}_{\perp}}{\beta}=-F \frac{\vec{\rho}^{\prime} \beta_{z}}{\beta}, \\
\gamma^{\prime} & =-\frac{F}{m_{\mu}} \sqrt{1+\left(\vec{\rho}^{\prime}\right)^{2}} .
\end{aligned}
$$

We avoid the derivation of the expressions $I_{ \pm}\left(\vec{p}_{\perp}, E, \vec{\rho}, z\right)$; instead, we use the canonical relationships shown in the Appendix, Eqs. (A6) and (A7), then

$$
I_{ \pm}^{\prime}=-F\left[\frac{1}{\beta} \vec{\rho}^{\prime} \frac{\partial \vec{\rho}}{\partial \psi_{ \pm}}-\frac{\partial t}{\partial \psi_{ \pm}} \sqrt{1+\left(\vec{\rho}^{\prime}\right)^{2}}\right]
$$

Thus, we can simply use the solutions in (3.27) and (3.37) in order to perform averaging over the phases $\psi_{ \pm}$. Note that

$$
\begin{aligned}
\vec{\rho}^{\prime} \frac{\partial \vec{\rho}}{\partial \psi_{ \pm}} & =\operatorname{Re}\left(\hat{x}^{\prime} \frac{\partial \hat{x}^{*}}{\partial \psi_{ \pm}}\right) \text {and } \\
\sqrt{1+\left(\vec{\rho}^{\prime}\right)^{2}} & \approx \sqrt{1+\kappa^{2}}+\frac{\kappa}{\sqrt{1+\kappa^{2}}}\left(k u_{1}+u_{2}^{\prime}\right) .
\end{aligned}
$$

The force $F$ has to be taken as shown in (4.12). The term on the right-hand side of the equations in (4.11) after averaging over $\psi_{ \pm}$becomes proportional to $a_{ \pm}^{2}$ [see Eq. (3.27)], i.e., the cooling decrements can be defined:

$$
\left\langle I_{ \pm}^{\prime}\right\rangle=-\Lambda_{ \pm} I_{ \pm},
$$

taking into account (3.35). Performing the averaging, we find

$$
\begin{aligned}
\frac{\Lambda_{ \pm}}{\Lambda_{o}}= & \pm \frac{1+\kappa^{2}}{\left(\alpha_{+}-\alpha_{-}\right) \alpha_{ \pm}}\left[2 \alpha_{ \pm}+\alpha_{ \pm}^{2}+Q_{ \pm}^{2}\right. \\
& \left.-\frac{\kappa^{2}}{1+\kappa^{2}}\left(1+\alpha_{ \pm}\right)^{2}\right] .
\end{aligned}
$$

Taking into account relationships in (3.28) and (3.29), we find the sum of transverse decrements:

$$
\frac{\Lambda_{+}+\Lambda_{-}}{\Lambda_{o}}=2-\frac{\kappa^{2}}{1+\kappa^{2}} \hat{D} .
$$

Combining all the three decrements, we find

$$
\begin{aligned}
\Lambda_{+}+\Lambda_{-}+\Lambda_{\gamma} & =2 \Lambda_{o}\left(\beta^{2}+\frac{1}{2} \frac{p}{\log } \frac{\partial \log }{\partial p}\right) \equiv \Lambda \\
& =2 \Lambda_{0} \hat{\beta}^{2},
\end{aligned}
$$

where we have introduced the parameter

$$
\hat{\beta}=\sqrt{\beta^{2}+\frac{1}{2} \frac{p}{\log } \frac{\partial \log }{\partial p}}=\sqrt{\beta^{2}+\frac{1-(\beta / \gamma)^{2}}{\log }},
$$

although in the following we will not distinguish between $\hat{\beta}$ and $\beta$, assuming

$$
\beta^{2} \gg \frac{1}{2} \frac{p}{\log } \frac{\partial \log }{\partial p} .
$$

This result (4.17) agrees with the dissipation theorem [28-30]:

$$
\sum_{\alpha=1}^{3} \Lambda_{\alpha}=-\left\langle\frac{1}{\beta_{z}} \frac{\partial}{\partial \vec{p}} \vec{F}(\vec{p}, \vec{r})\right\rangle .
$$

The distribution of the two transverse cooling rates is characterized by the difference $\Lambda_{+}-\Lambda_{-}$. Using the definitions $\alpha_{ \pm}$, etc., we obtain

$$
\frac{\Lambda_{+}-\Lambda_{-}}{\Lambda_{o}}=\frac{1}{\sqrt{R^{2}-G}}\left[q^{2}-1+\kappa^{2}(R \hat{D}-1)\right] \frac{1}{1+\kappa^{2}} .
$$

\section{Equating the cooling decrements}

If the three cooling decrements are equal,

$$
\Lambda_{\gamma}=\Lambda_{+}=\Lambda_{-}=\Lambda / 3
$$

then

$$
\hat{D}=2 \frac{1+\kappa^{2}}{\kappa^{2}}\left(1-\frac{2}{3} \beta^{2}\right)
$$

and

$$
q \equiv \frac{k_{c}}{k}-1=\beta \sqrt{\frac{1+\kappa^{2}}{3-\beta^{2}}} .
$$

As follows from Eq. (3.14), conditions (4.20) and (4.21) also determine the effective field index $g$ as a function of $\kappa$ and $\beta$ :

$$
g=-\frac{1-\kappa^{2}}{1+\kappa^{2}} \sqrt{\frac{1+\kappa^{2}}{3-\beta^{2}}}+\frac{\kappa^{2}}{2\left(1+\kappa^{2}\right)} \frac{4 \beta^{2}-3}{3-2 \beta^{2}} .
$$

Condition (4.21) indicates the necessity of sufficiently strong solenoidal and dipole fields according to 
Eqs. (3.11) or (3.13), while condition (4.22) determines the quadrupole strength.

The balanced cooling area in terms of parameters $\kappa$ and $\beta$ can be limited by the dynamical stability condition (3.24), which can be rewritten as

$$
\begin{aligned}
0 & <\left[\beta\left(\frac{1+\kappa^{2}}{3-\beta^{2}}\right)^{1 / 2}+\kappa^{2} \frac{\frac{3}{4}-\beta^{2}}{3-2 \beta^{2}}\right] \\
& <\left(\frac{\left(1+\kappa^{2}\right)^{2}}{4 \kappa^{2}} \frac{\left(1-\frac{2}{3} \beta^{2}\right)}{\left(1-\frac{1}{3} \beta^{2}\right)^{2}}\right) .
\end{aligned}
$$

In the region $\beta^{2}<3 / 4$, the periodic orbit seems to appear stable at any $\kappa$ value, although for $\beta^{2}>3 / 4$ condition (4.21) also leaves the beam stable in a wide range of $\kappa<$ 1. Note, however, that if $\kappa$ is too small then the beam stability is worse, as can be seen from the formula for the oscillation tune $Q_{-}$,

$$
Q_{-} \approx \sqrt{G / 2 R} \approx \kappa\left(\frac{\sqrt{3-\beta^{2}}}{3-2 \beta^{2}} \beta\right)^{1 / 2}
$$

\section{EQUILIBRIUM EMITTANCES}

\section{A. Scattering and straggling}

Besides the average ionization energy loss when moving through the absorber, each muon exchanges momentum with the atoms of the absorber, both in direction and magnitude. The average angular scattering from absorber nuclei and electrons is

$$
\frac{d \theta_{s c}^{2}}{d z}=\frac{Z+1}{\gamma \beta^{2}} \frac{m_{e}}{m_{\mu}} \Lambda .
$$

The spread about the average energy loss is effectively only caused by collisions with electrons, because of the large nucleon mass relative to the electron mass:

$$
\frac{d}{d z}(\delta \gamma)^{2}=\frac{\gamma \beta^{2}}{4 \log }\left(\gamma^{2}+1\right) \frac{m_{e}}{m_{\mu}} \Lambda .
$$

It is well known that the momentum or energy spread does not include the factor of the Coulomb log since it is determined characteristically by interactions with the maximum momentum transfer. The contribution of low momentum transfer collisions to energy diffusion appears insignificant, contributing at most to angular scattering and energy loss. Correspondingly, the energy straggling grows rapidly with the Lorentz factor of the muon beam.

\section{B. Longitudinal equilibrium emittance, energy spread, and bunch length}

The equilibrium synchrotron emittance results from the balance between damping and growth rates due to both energy straggling and angle scattering. In the vicinity of the reference orbit, the adiabatic invariant can be represented as a quadratic function of energy deviation from the equilibrium value and reference time $\tau$ as shown in (3.56). Then the diffusion growth rate of $I_{S}$ can generally be found as

$$
\left(I_{S}^{\prime}\right)_{d}=\frac{\eta}{2 \omega Q_{S}} \frac{d}{d z}(\delta \gamma)^{2}+\frac{\omega Q_{S}}{2 \eta} \frac{d}{d z}(\delta \hat{t})^{2},
$$

where the straggling rate is given by Eq. (5.2). The diffusion rate of $\hat{t}$ can be found using Eq. (3.38) taking into account the continuity of the total phase, $\omega t-k_{\mathrm{rf}} z$, in a collision,

$$
\frac{d}{d z}(\delta \hat{t})_{s c}^{2}=\frac{D^{2}}{2 \beta^{2}\left(1+\kappa^{2}\right)} \frac{d}{d z} \theta_{s c}^{2},
$$

with the angle scattering rate as given by (5.1).

Applying the Langevin balance equation [31],

$$
I_{S}^{\prime}=-\Lambda_{\gamma} I_{S}+\left(I_{S}^{\prime}\right)_{d}
$$

we obtain the normalized equilibrium synchrotron emittance:

$$
\begin{aligned}
\varepsilon_{S} & \equiv\left\langle I_{S}\right\rangle_{e q}=\left(I_{S}^{\prime}\right)_{d} / \Lambda_{\gamma} \\
& =\frac{\Lambda}{4 \Lambda_{\gamma}} \frac{m_{e}}{m_{\mu}} \gamma \beta^{2}\left[\frac{\eta}{\omega Q_{S}} \frac{\gamma^{2}+1}{2 \log }+\frac{Z+1}{\gamma^{2} \beta^{6}} \frac{\omega Q_{S}}{\eta k^{2}} \frac{\hat{D}^{2} \kappa^{2}}{1+\kappa^{2}}\right] .
\end{aligned}
$$

The first term in the brackets in (5.5) corresponds to energy straggling and the second term is due to $\omega t$ diffusion due to scattering.

Now, knowing the equilibrium emittance, one can easily determine the equilibrium energy spread and bunch length, relying on Eq. (3.56):

$$
\left\langle(\Delta \gamma)^{2}\right\rangle=\frac{\omega Q_{S}}{\eta} \varepsilon_{S} \quad \text { and } \quad\left\langle\tau^{2}\right\rangle=\frac{\eta}{\omega Q_{S}} \varepsilon_{S} .
$$

\section{Equilibrium transverse emittances}

In order to calculate the scattering rates of transverse emittances, one can use the general expressions (3.33)(3.35) and (3.40), where the helix radius $a$ is a function of total momentum $p$. Both the angle and energy scattering will contribute to the growth of transverse amplitudes. Calculating the growth rate due to momentum jumps along the $x_{2}$ direction, we have to take into account that this axis is not perpendicular to the particle total momentum direction, but makes an angle with it whose tangent is $\kappa=$ $p_{\perp} / p_{z}$; thus we find

$$
\begin{aligned}
k^{2}\left(a_{ \pm}^{2}\right)_{s c}^{\prime}= & \frac{1 /\left(1+\kappa^{2}\right)}{\left(\alpha_{+}-\alpha_{-}\right)^{2}}\left\{\frac{1}{2}\left[1+\frac{Q_{ \pm}^{2}}{\alpha_{ \pm}^{2}}\left(1+\kappa^{2}\right)^{3}\right] \frac{d \theta_{s c}^{2}}{d z}\right. \\
& \left.+\frac{\kappa^{2}}{\gamma^{2} \beta^{4}}\left[1+\sqrt{1+\kappa^{2}} \hat{D} \alpha_{\mp}\right]^{2} \frac{d}{d z}(\delta \gamma)^{2}\right\} .
\end{aligned}
$$


Applying Langevin's balance equations

$$
a_{ \pm}^{2}=-\Lambda_{ \pm} a_{ \pm}^{2}+\left(a_{ \pm}^{2}\right)_{s c}^{\prime}
$$

we find the following expressions for the equilibrium normalized transverse emittances:

$$
\left\langle I_{ \pm}\right\rangle=\varepsilon_{ \pm}=\frac{\Gamma_{ \pm}}{4 k Q_{ \pm}} \frac{m_{e}}{m_{\mu} \beta} \frac{\Lambda / \Lambda_{ \pm}}{\left(1+\kappa^{2}\right)^{5 / 2}}
$$

where

$$
\Gamma_{ \pm} \equiv \frac{(Z+1)\left[\alpha_{ \pm}^{2}+\left(1+\kappa^{2}\right)^{3} Q_{ \pm}^{2}\right]+\kappa^{2}\left[\alpha_{ \pm}-\left(1+\kappa^{2}\right)^{3 / 2}\right]^{2}\left(\gamma^{2}+1\right) / 2 \log }{\alpha_{ \pm}^{2}+\left(1+\kappa^{2}\right) \hat{D}^{-1}}
$$

\section{NUMERICAL EXAMPLE OF A HELICAL CHANNEL}

Table I shows a numerical example of a helical cooling channel using the equations derived in earlier sections. The beam momentum of $100 \mathrm{MeV} / c$ is low compared to the $200 \mathrm{MeV} / c$ of earlier studies in order to attain the best transverse and longitudinal equilibrium emittances as well as to shorten the beam path and reduce the integrated energy loss in the absorber. A rather tight helix $(\kappa \approx 1)$ has been chosen in this calculation to moderate the dispersion required for balanced cooling. Lower dispersion improves beam stability and reduces the contribution of energy spread to the radial beam size, the straggling contribution to equilibrium transverse emittances due to dispersion (to about 6\%), and the angle scattering contribution to the longitudinal emittance (to 33\%). Note that the calculated $6 \mathrm{D}$ emittance may not be a minimum, although

TABLE I. Estimated parameters of a helical 6D cooling channel.

\begin{tabular}{lcccc}
\hline \hline \multicolumn{1}{c}{ Parameter } & Unit & Initial & Middle $^{\mathrm{d}}$ & Final $^{\mathrm{c}}$ \\
\hline Beam momentum, $p^{\mathrm{a}}$ & $\mathrm{MeV} / \mathrm{c}$ & 100 & 100 & 100 \\
Solenoid field, $B$ & $\mathrm{~T}$ & 3.5 & 8 & 14 \\
Cyclotron wavelength, $\lambda_{c}=2 \pi / k_{c}{ }^{\mathrm{a}}$ & $\mathrm{m}$ & 0.60 & 0.26 & 0.14 \\
Helix period, $\lambda=2 \pi / k$ & $\mathrm{~m}$ & 1 & 0.44 & 0.22 \\
Helical magnet inner radius & $\mathrm{cm}$ & 30 & 12 & 7 \\
Transverse field at magnet coil & $\mathrm{T}$ & 1.7 & 4.2 & 7.0 \\
Transverse field at beam center & $\mathrm{T}$ & 0.7 & 1.6 & 3.0 \\
Helix quadrupole gradient & $\mathrm{T} / \mathrm{m}$ & 1.2 & 7.5 & 20 \\
Helix orbit radius, $a^{\mathrm{a}}$ & $\mathrm{cm}$ & 15 & 6 & 3 \\
Dispersion, $D$ & $\mathrm{~cm}$ & 37 & 15 & 7.5 \\
Transverse tunes, $Q_{+} / Q_{-}$ & & $0.94 / 0.57$ & $0.94 / 0.57$ & $0.94 / 0.57$ \\
Transverse beta functions, $\beta_{+} / \beta_{-}$ & $\mathrm{cm}$ & $16 / 26$ & $6 / 10$ & $3.2 / 5.2$ \\
Accelerating rf field amplitude & $\mathrm{MV} / \mathrm{m}$ & 40 & 40 & 40 \\
Frequency, $f=\omega / 2 \pi$ & $\mathrm{GHz}$ & 0.2 & 0.8 & 1.6 \\
Absorber energy loss rate $d E / d s$ & $\mathrm{MeV} / \mathrm{m}$ & 14 & 14 & 14 \\
6D cooling decrement length, $\Lambda^{-1}$ & $\mathrm{~m}$ & 4 & 4 & 4 \\
Individual decrement lengths & $\mathrm{m}$ & 12 & 12 & 12 \\
Synchrotron beta function, $\beta_{s}$ & $\mathrm{~m}$ & 0.95 & 0.47 & 0.32 \\
Synchrotron tune, $Q_{s}=1 / \omega \beta_{s}$ & & 0.25 & 0.12 & 0.08 \\
Synchrotron admittance, $I_{a}$ & $\mathrm{~cm}$ & 3.0 & 0.14 \\
Synchrotron emittance, $\varepsilon_{S}$ & $\mathrm{~cm}$ & $1.5^{\mathrm{b}}$ & 0.37 & $3 \times 10^{-2 \mathrm{c}}$ \\
Relative momentum spread & $\%$ & $7.5^{\mathrm{b}}$ & $2^{\mathrm{b}}$ & $1.1^{\mathrm{c}}$ \\
Bunch length & $\mathrm{cm}$ & $30^{\mathrm{b}}$ & $7.5^{\mathrm{b}}$ & $0.15^{\mathrm{c}}$ \\
Beam width, $\Delta a$ & $\mathrm{~cm}$ & $1.7 / 1.7^{\mathrm{b}}$ & $0.56^{\mathrm{b}}$ & $(1 / 3) \times 10^{-2 \mathrm{c}}$ \\
Transverse emittances, $\varepsilon_{+} / \varepsilon_{-}$ & $\mathrm{cm}$ rad & $1.8 / 1.5^{\mathrm{b}}$ & $0.45 / 0.28^{\mathrm{b}}$ \\
Beam widths, $\sigma_{1} / \sigma_{2}$ & $\mathrm{~cm}$ & & \\
\hline \hline
\end{tabular}

${ }^{\mathrm{a}}$ Reference orbit.

${ }^{\mathrm{b}}$ Maximum deviation from reference orbit.

${ }^{c}$ rms equilibrium value.

${ }^{\mathrm{d}}$ At the beginning of the $0.8 \mathrm{GHz}$ section. 
further optimization has yet to improve the estimated equilibrium values significantly. The cooling effect in this calculation in terms of reduction of the $6 \mathrm{D}$ emittance is $5 \times 10^{5}$. The total energy loss in the absorber is about $1.12 \mathrm{GeV}$. For a channel of continuous dense hydrogen gas with $14 \mathrm{MeV} / \mathrm{m}$ of energy loss, this implies a $6 \mathrm{D}$ cooling channel length, $L=\frac{1.12}{0.014} / \sqrt{1+\kappa^{2}}=56 \mathrm{~m}$.

\section{DISCUSSION}

The initial low momentum muon beam with relatively small momentum spread (7.5\%) in the example above could be obtained using a short helical channel with an absorber but no rf. This possibility was proposed and treated previously [17]. Using emittance exchange in this precooling helical channel, all the cooling power of the absorber (with or without wedges) can be taken from the transverse coordinates and concentrated on the longitudinal one to cool the energy spread. Maintaining the transverse emittances, or even by allowing some increase in them for more reduction of energy spread, it is possible to decrease the absolute energy spread a factor of 10 or more before the bunch length is increased significantly. Then, the beam can be captured and bunched by a rf field and injected into the basic cooling channel described by Table I. In this way, the total 6D emittance reduction factor could exceed $10^{6}$. The estimated length of such a deceleration and capture section is less than $25 \mathrm{~m}$.

The example channel assumes smaller, higherfrequency rf cavities will be used when the beam dimensions have been cooled enough to allow them. Reduced transverse dimensions imply that the desired strength of the magnetic fields would be easier to achieve. Higher frequencies make it easier to achieve the desired rf gradients. Short adiabatic helical transition sections would be used to match one stage to the next.

In order to optimize the rf cavity acceptance, each cavity axis could be centered on and aligned with the periodic orbit as the beam wound around the axis of the solenoid, perhaps with the rf waveguides passing through the gap in the helical dipole coil. The large aperture magnets and $\mathrm{rf}$ cavities for the $200 \mathrm{MHz}$ stage may be a serious technical challenge and future studies will be toward replacing the initial stage with precooling sections to reduce the beam size. The precooling under consideration involves transverse cooling with a helical quadrupole section and longitudinal cooling in a short helical dipole section without rf.

The estimated cooling channel length of $56 \mathrm{~m}$ for a bunched muon beam of $100 \mathrm{MeV} / c$ assumes that the channel contains rf cavities that operate while filled with dense hydrogen gas. At $50 \mathrm{~atm}$ and $77 \mathrm{~K}$, hydrogen gas density is about $21.5 \%$ of liquid hydrogen and the corresponding $d E / d s$ for muons with $100 \mathrm{MeV} / c$ momentum, or $46 \mathrm{MeV}$ kinetic energy, is about $14 \mathrm{MeV} / \mathrm{m}$. In this case, the rf cavities must provide sufficient gradient to compensate for $\frac{d E}{d z}=\frac{d E}{d s} \sqrt{1+\kappa^{2}}=20 \mathrm{MeV} / \mathrm{m}$ energy loss and provide sufficient rf bucket area for longitudinal beam stability. Thus an average accelerating gradient of around $40 \mathrm{MeV} / \mathrm{m}$ is required.

The project by Muons, Inc. and IIT presently underway at Fermilab to develop high-gradient pressurized rf cavities is designed to explore the use of hydrogen and helium gas up to more than $100 \mathrm{~atm}$ pressure at temperature down to $77 \mathrm{~K}$. Surface gradients of $80 \mathrm{MV} / \mathrm{m}$ for stable operation have been achieved at $800 \mathrm{MHz}$ with $20 \mu$ s pulses in hydrogen at $17 \mathrm{~atm}$ at liquid nitrogen temperature using molybdenum electrodes. Scaling from the measured Paschen curve data from Lab G, hydrogen gas itself at $50 \mathrm{~atm}$ and $77 \mathrm{~K}$ will support gradients up to $330 \mathrm{MV} / \mathrm{m}$. Future proof-of-principle tests include operation in strong magnetic fields and in intense ionizing radiation. Providing sufficient power is also a necessary condition for highgradient cavities. A scheme has been investigated that implies that $50 \mathrm{MV} / \mathrm{m}$ could be generated at $200 \mathrm{MHz}$ using cold copper pillbox cavities for the short pulses required for a neutrino factory or muon collider using pulse compression techniques [32,33].

A cooling channel based on the use of helical magnets and a continuous homogeneous absorber offers advantages compared to other designs. One important advantage is that the cooling system can be described relatively simply with a time-independent, beam-path-independent Hamiltonian that does not depend on $z$. Thus the stability and evolution of the beam as it cools can be understood using wellestablished analytical linear and perturbative nonlinear techniques. The next steps in the development of the concepts presented here include simulations to verify the linear and nonlinear aspects of the beam dynamics and to continue the experimental investigations to the point that a complete, realistic 6D cooling channel can be designed, prototyped, and built. This concept and particular example are being developed and simulated by Muons, Inc. and the Thomas Jefferson National Accelerator Facility [34]. A description of the project and a preliminary exposition of the ideas presented in this paper were first reported in [35].

\section{CONCLUSIONS}

A magnetic channel filled with continuous absorber without special edge shaping can be used for emittance exchange cooling of the $6 \mathrm{D}$ emittance of a muon beam. This is true for any magnetic arrangement where higher momentum corresponds to a longer path length in a homogeneous absorber and therefore larger ionization energy loss.

The dynamical properties of an attractive example of such a channel have been investigated in some detail, where a solenoidal field is combined with a helical field to provide superior $6 \mathrm{D}$ cooling. The continuous nature of the magnetic fields and their helical invariance allows periodic orbits to be found and their stability and cooling properties to be completely analyzed. We have shown that 
a continuous, homogenous absorber using high-gradient hydrogen-gas-filled rf cavities could provide exceptional $6 \mathrm{D}$ cooling in a rather short channel.

Six-dimensional cooling using a homogeneous absorber may be a way to achieve the beam brightness needed for a muon collider. If engineering studies and simulations bear out this prediction, the case for a muon collider as a future energy frontier machine will be strengthened.

Recent discoveries have made a neutrino factory an attractive physics opportunity. The 6D cooling suggested here could provide a neutrino factory with superior performance and reduced costs.

\section{ACKNOWLEDGMENTS}

We are pleased to thank Dr. Boyce, Dr. Chattopadhyay, Dr. Dylla, Dr. Leemann, and Dr. Merminga of Thomas Jefferson National Accelerator Facility for the opportunity to continue this research and to investigate with simulations and further analytic calculations the validity of the proposals suggested here. We thank Dr. A. Bogacz and Dr. K. Yonehara for helpful comments. We also thank S. Derbeneva and L. Even for help in preparing the manuscript. Thomas Jefferson National Accelerator Facility is operated by the Southeastern Universities Research Association, Inc. under U.S. Department of Energy Contract No. DE-AC05-84ER40150. Muons, Inc. is supported in part by SBIR Grant No. DE-FG02-03ER83722.

\section{APPENDIX: HAMILTONIAN FRAMEWORK AND CANONICAL RELATIONSHIPS}

Hamilton's method provides some important relationships of beam dynamics in a magnetic field (or a stationary electromagnetic field). It is based on the introduction of a Hamiltonian with corresponding equations of motion. An ordinary Hamiltonian form is the energy function

$$
\hat{E}=\sqrt{p^{2}+m^{2}}+A_{o}=\sqrt{(\vec{P}-\vec{A})^{2}+m^{2}}+A_{o},
$$

with equations of motion

$$
\dot{\vec{P}}=-\frac{\partial}{\partial \vec{r}} \hat{E}(\vec{P}, \vec{r}, t)
$$

and

$$
\dot{\vec{r}}=\frac{\partial}{\partial \vec{P}} \hat{E}
$$

In the case of a particle beam transported along a fixed direction $z$, it is convenient to consider $z$ as a time argument, while the time $t$ can be treated as one of three independent coordinates $x, y, t$. Hamilton's function and equations of motion in this representation can be quickly derived using the covariant equation for the wave function $\Psi(\vec{r}, t)$, or the relativistic Schroedinger equation

$$
\left[\left(\vec{E}+A_{o}\right)^{2}-(\hat{\vec{P}}+\vec{A})^{2}-m^{2}\right] \Psi=0,
$$

where

$$
\hat{E}=i \hbar \frac{\partial}{\partial t} \quad \text { and } \quad \vec{P}=-i \hbar \vec{\nabla}
$$

are the time and space components of 4-vector momentum as a quantum operator. In the quasiclassical limit, this equation can be rewritten, optionally, in two possible forms:

$$
i \hbar \frac{\partial}{\partial t} \Psi=\left[\sqrt{(\hat{\vec{P}}+\vec{A})^{2}+m^{2}}-A_{o}\right] \Psi \equiv H_{t} \Psi,
$$

with equations of motion

$$
\dot{\bar{P}}=-\frac{1}{i \hbar}\left[H_{t}, \vec{P}\right]=\left\{H_{t}, \vec{P}\right\} \rightarrow-\frac{\partial H_{t}}{\partial \vec{r}},
$$

and

$$
\dot{\vec{r}}=\frac{\partial H_{t}}{\partial \vec{P}}
$$

or

$$
\begin{aligned}
i \hbar \frac{\partial}{\partial z} \Psi & =-\left[\sqrt{\left(\hat{E}+A_{o}\right)^{2}-\left(\vec{P}_{\perp}+\vec{A}_{\perp}\right)^{2}-m^{2}}+A_{z}\right] \Psi \\
& \equiv H_{z}\left(\vec{P}_{\perp}, \vec{\rho}, \hat{E}, t, z\right) \Psi
\end{aligned}
$$

with equations of motion

$$
\vec{P}_{ \pm}^{\prime}=-\frac{\partial}{\partial \vec{\rho}} H_{z}
$$

and

$$
\begin{gathered}
\vec{\rho}^{\prime}=\frac{\partial}{\partial \vec{P}_{\perp}} H_{z} \\
\hat{E}^{\prime}=-\frac{1}{i \hbar}\left[H_{z}, \hat{E}\right]=\frac{\partial H_{z}}{\partial t}, \quad t^{\prime}=-\frac{\partial}{\partial \hat{E}} H_{z} .
\end{gathered}
$$

Thus, in the $z$ representation Hamilton's function coincides with the canonical momentum (with reversed sign) $P_{z}=p_{z}+A_{z}$ taken as a function of energy and transverse momentum according to the covariant expression $E^{2}-$ $p^{2}=m^{2}$.

For a helical structure, it is convenient to consider particle dynamics in terms of a helical frame $\left(x_{1}, x_{2}, z\right)$, with

$$
x_{1}+i x_{2}=(x+i y) e^{-i k z} .
$$

The new Hamiltonian and equations of motion can be simply found using the wave equation in (A1), taking into account that

$$
\vec{\nabla}_{\perp} \equiv \vec{e}_{x} \frac{\partial}{\partial x}+\vec{e}_{y} \frac{\partial}{\partial y}=\vec{e}_{1} \frac{\partial}{\partial x_{1}}+\vec{e}_{2} \frac{\partial}{\partial x_{2}}
$$

and

$$
\vec{P}_{\perp}=\vec{e}_{1} P_{1}+\vec{e}_{2} P_{2}
$$


while

$$
\frac{\partial}{\partial z} \Psi(x, y, z)=\left(\frac{\partial}{\partial z}-k x_{1} \frac{\partial}{\partial x_{2}}+k x_{2} \frac{\partial}{\partial x_{1}}\right) \Psi\left(x_{1}, x_{2}, z\right) .
$$

Thus, the new Hamiltonian is

$$
\begin{aligned}
H_{h}= & -\sqrt{\left(\hat{E}+A_{o}\right)^{2}-m^{2}-\left(P_{1}+A_{1}\right)^{2}-\left(P_{2}+A_{2}\right)^{2}} \\
& +A_{z}+k\left(x_{2} P_{1}-x_{1} P_{2}\right) .
\end{aligned}
$$

This is the so-called helical invariant. Since the components of the vector potential $A_{1}, A_{2}, A_{z}$ are functions of only $x_{1}, x_{2}$ (i.e., $\rho$ and $\psi \equiv \varphi-k z$ ), the Hamiltonian $H_{h}$ is conserved for any particle trajectory, together with the energy $E$ (assuming no rf and space charge forces).

Solving the equations of motion in a helical structure, one can find the generalized phases $\Psi_{ \pm}$and adiabatic invariants (quantum numbers) $I_{ \pm}$as functions of $x_{1}, P_{1} ; x_{2}, P_{2} ; E$. In these variables, the Hamiltonian (A3) is a function of only $I_{+}, I_{-}$. and $E$ :

$$
I_{ \pm}^{\prime}=\frac{\partial}{\partial \psi_{ \pm}} H_{h}\left(I_{+}, I_{-}, E\right)=0
$$

and

$$
\psi_{ \pm}^{\prime}=\frac{\partial}{\partial I_{ \pm}} H_{h}\left(E, I_{+}, I_{-}\right)=k Q_{ \pm}\left(E, I_{+}, I_{-}\right)=\text {const. }
$$

The energy $E$, being a global invariant in a magnetic field, is not redefined, but there should appear a new time variable $\hat{t}$ as a canonical phase with a constant rate,

$$
\hat{t}^{\prime}=-\frac{\partial}{\partial E} H_{h}\left(E, I_{+}, I_{-}\right)=\text {const, }
$$

while the time $t$ on a particle trajectory can be considered as a function of all the new variables including $\hat{t}$ and $E$. There is a set of differential relationships between the new and old canonical variables [36]. For our situation, the important relationships are

$$
\frac{\partial I_{ \pm}}{\partial \bar{P}_{\perp}}=\frac{\partial \bar{P}}{\partial \psi_{ \pm}}
$$

and

$$
\frac{\partial I_{ \pm}}{\partial E}=-\frac{\partial t}{\partial \psi_{ \pm}} .
$$

These can be simply proved comparing the Poisson brackets $\left\{I_{ \pm}, \vec{\rho}\right\}$ and $\left\{I_{ \pm}, t\right\}$ in terms of old and new variables.

In order to find the dynamical sense of $\hat{t}$, one has to integrate the equation

$$
t^{\prime}=\frac{1}{\beta_{z}}=\frac{\sqrt{1+\left(\vec{\rho}^{\prime}\right)^{2}}}{\beta}
$$

along a solved particle trajectory. The inverse particle velocity $\beta_{z}^{-1}$ can be represented as

$$
\beta_{z}^{-1}=\left\langle\beta_{z}^{-1}\right\rangle+\tilde{\beta}_{z}^{-1},
$$

where $\tilde{\beta}_{z}^{-1}$ oscillates as a function of $\psi_{+}, \psi_{-}$:

$$
\left\langle\tilde{\beta}_{z}^{-1}\right\rangle=0 .
$$

Note that $\left\langle\beta_{z}^{-1}\right\rangle=1 /\left\langle\beta_{z}\right\rangle$.

If we introduce the waving fraction of time $\tilde{t}$ as

$$
t=\langle t\rangle+\tilde{t}\left(\psi_{+}, \psi_{-}\right), \quad\langle\tilde{t}\rangle=0,
$$

where

$$
\tilde{\beta}_{z}^{-1}=\tilde{t}^{\prime}=k\left(Q_{+} \frac{\partial}{\partial \Psi_{+}}+Q_{-} \frac{\partial}{\partial \Psi_{-}}\right) \tilde{t},
$$

then the variable $\hat{t}$ can be identified as

$$
\hat{t}=t-\tilde{t}\left(\psi_{+}, \psi_{-}\right) .
$$

This can be proved by comparing Eq. (A5) to the equation for the original time:

$$
\begin{aligned}
t^{\prime} & =-\left(\frac{\partial}{\partial E}+\frac{\partial I_{+}}{\partial E} \frac{\partial}{\partial I_{+}}+\frac{\partial I_{-}}{\partial E} \frac{\partial}{\partial I_{-}}\right) H_{h}\left(E, I_{+}, I_{-}\right) \\
& =\hat{t}^{\prime}+k Q_{+} \frac{\partial t}{\partial \psi_{+}}+k Q_{-} \frac{\partial t}{\partial \psi_{-}} .
\end{aligned}
$$

Note the important relationships which follow from Eqs. (A4) and (A5):

$$
\frac{\partial}{\partial I_{ \pm}} \frac{1}{\left\langle\beta_{z}\right\rangle}=-k \frac{\partial}{\partial E} Q_{ \pm} .
$$

These show that the so-called slippage factors, i.e., emittance-related dispersion of the translation velocity $\left\langle\beta_{z}\right\rangle$, are simply proportional to the chromaticity of particle tunes $[20,21]$.

To determine the time $t$ as function of new variables $\left(a_{ \pm}, \psi_{ \pm}\right)$, one has to integrate the equation

$$
t^{\prime}=\frac{1}{\beta_{z}}=\frac{1}{\beta} \sqrt{1+\left|\hat{x}^{\prime}+i k \hat{x}\right|^{2}}
$$

along a solved particle trajectory. In linear approximation for free particle oscillations near the helical orbit $\vec{a}(p, z)$, we have

$$
t^{\prime} \approx \frac{\sqrt{1+\kappa^{2}}}{\beta}+\frac{\kappa\left(k u_{1}+u_{2}^{\prime}\right)}{\beta \sqrt{1+\kappa^{2}}} .
$$

To integrate $u_{1}$, we have to substitute $u_{1}$ as the solution shown in (3.30):

$$
\begin{aligned}
\int d z \cdot u_{1} & =\int\left(a_{+} \cos \psi_{+}+a_{-} \cos \psi_{-}\right) d z \\
& =\frac{a_{+}}{k Q_{+}} \sin \psi_{+}+\frac{a_{-}}{k Q_{-}} \sin \psi_{-} .
\end{aligned}
$$


Then we obtain Eq. (3.37). The derivatives $\frac{\partial a_{ \pm}^{2}}{\partial E}$ can be found using the relationships in (3.31)-(3.34), in which the variables of the rotating frame $\left(x_{1}, x_{1}^{\prime}, x_{2}, x_{2}^{\prime}\right)$ are to be considered as functions of $\left(x, p_{x}\right),\left(y, p_{y}\right)$, and energy $E$ :

$$
\begin{gathered}
\hat{x} \equiv x_{1}+i x_{2}=(x+i y) e^{-i k z}, \\
\hat{x}^{\prime} \equiv x_{1}^{\prime}+i x_{2}^{\prime} \\
=-i k(x+i y) e^{-i k z}+\frac{\left(p_{x}+i p_{y}\right) e^{-i k z}}{\sqrt{E^{2}-m^{2}-\left|p_{x}+i p_{y}\right|^{2}}} .
\end{gathered}
$$

Thus, we find

$$
\frac{\partial \hat{x}}{\partial E}=0 \quad \text { and } \quad \frac{\partial \hat{x}^{\prime}}{\partial E}=-\frac{\hat{x}^{\prime}+i k \hat{x}}{p_{z}^{2}} E .
$$

In first approximation, if we neglect the deviations off the equilibrium orbit on the right-hand equation in (A9), then

$$
\frac{\partial x_{1}^{\prime}}{\partial E}=0, \quad \frac{\partial x_{2}^{\prime}}{\partial E}=-\frac{k a}{p_{z}^{2}} E=-\frac{k a}{E \beta^{2}}\left(1+\kappa^{2}\right) .
$$

Now, we can take the derivatives

$$
\begin{aligned}
\frac{\partial}{\partial E} a_{ \pm}^{2} & =2 \frac{x_{2}^{\prime}-\alpha_{\mp} k\left(x_{1}-a\right)}{\left(\alpha_{+}-\alpha_{-}\right)^{2}}\left[-\frac{k a}{E \beta^{2}}\left(1+\kappa^{2}\right)+\alpha_{ \pm} \frac{k d a}{\beta d p}\right] \\
& = \pm \frac{2 a}{E \beta^{2}} \frac{\left[\hat{D} \alpha_{\mp}-1-\kappa^{2}\right]}{\alpha_{+}-\alpha_{-}} a_{ \pm} \cos \psi_{ \pm} .
\end{aligned}
$$

Finally, using the relationship in (3.28), we find Eq. (3.39).

[1] Muon Collaboration, M. M. Alsharo'a et al., Phys. Rev. ST Accel. Beams 6, 081001 (2003).

[2] A. N. Skrinsky, in Proceedings of the 2002 Joint USPASCAS-Japan-Russia Accelerator School, Long Beach, CA, 2002, edited by Helmut Wiedemann, Daniel Brandt, Eugene A. Perevedentsev, and Shin-ichi Kurokawa (World Scientific, Singapore, 2004).

[3] Muon Collider Collaboration, C. M. Ankenbrandt et al., Phys. Rev. ST Accel. Beams 2, 081001 (1999).

[4] S. Geer, Phys. Rev. D 57, 6989 (1998).

[5] Feasibility Study on a Neutrino Source Based on a Muon Storage Ring, edited by D. Finley and N. Holtkamp, http:// www.fnal.gov/projects/muon_collider/nu-factory/ fermi_study_after_aprillst/

[6] BNL Report No. BNL-52623, 2001, edited by S. Ozaki, R. Palmer, M. Zisman, and J. Gallardo, http://www.cap.bnl.gov/mumu/studyii/FS2-report.html

[7] A. N. Skrinsky and V. V. Parkhomchuk, Sov. J. Part. Nuclei 12, 223 (1981).

[8] D. M. Kaplan, in Proceedings of Snowmass 2001, Snowmass Village, CO, http://www.slac.stanford.edu/ econf/C010630/papers/M102.PDF
[9] Invented by Y.S. Derbenev and A. Kondratenko at the Institute of Nuclear Physics in Novosibirsk, the Siberian snake is a device to be inserted into the lattice of a circular accelerator to preserve the polarization of a proton beam during the acceleration process. See [22] below.

[10] R. P. Johnson et al., in First International Workshop on Hydrogen in Materials and Vacuum Systems, edited by G. Myneni and S. Chattopadhyay, AIP Conf. Proc. No. 671 (AIP, New York, 2003), pp. 328-335.

[11] Rolland Johnson and Daniel Kaplan, DOE STTR Proposal No. 70255B0I-I, 2002.

[12] R.E. Hartline, R.P. Johnson, M. Kuchnir, C.M. Ankenbrandt, A. Moretti, M. Popovic, D. M. Kaplan, and K. Yonehara, http://www-mucool.fnal.gov/monotes/ public/pdf/muc0285/muc0285.pdf

[13] Sanborn C. Brown, Basic Data of Plasma Physics, The Fundamental Data on Electrical Discharges in Gases, American Vacuum Society Classics (AIP, New York, 1993), p. 149.

[14] MICE Collaboration, G. Gregoire et al., http://hep04.phys.iit.edu/cooldemo/micenotes/public/pdf/MICE0021/ MICE0021.pdf

[15] http://www.muonsinc.com/

[16] http://wwwasd.web.cern.ch/wwwasd/geant4/geant4.html

[17] Y. Derbenev, http://www-mucool.fnal.gov/mcnotes/ public/ps/muc0108/muc0108.ps.gz

[18] V. Balbekov, http://www-mucool.fnal.gov/mcnotes/public/ps/muc0246/muc0246.ps.gz

[19] Al Garren, Harold Kirk, and Steve Kahn, http://www.fnal.gov/projects/muon_collider/eexchange/workshop03/garren.pdf; see also http://www.physics.ucla.edu/hep/mc/ tucson/rc2003.htm

[20] Steve Kahn, Alper Garren, and Harold Kirk, http:// www.fnal.gov/projects/muon_collider/eexchange/workshop03/kahn1.pdf

[21] M. Syphers E. Courant, W. Fischer, A. Luccio, F. Mariam, S. Peggs, F. Pilat, T. Roser, S. Tepikian, N. Tsoupas, E. Willen, T. Katayama, K. Hatanaka, T. Kawaguchi, M. Okamura, T. Tominaka, H. Wu, V. Ptitsin, and Y. Shatunov, in Proceedings of the 1997 Particle Accelerator Conference, Vancouver, Canada (IEEE, Piscataway, NJ, 1997).

[22] Ya. S. Derbenev and A. M. Kondratenko, in High Energy Physics with Polarized Beams and Polarized Targets, edited by G. H. Thomas, AIP Conf. Proc. No. 51 (AIP, New York, 1979), p. 292.

[23] E. Willen, M. Anerella, J. Escallier, G. Ganetis, A. Ghosh, R. Gupta, M. Harrison, A. Jain, W. MacKay, A. Marone, J. Muratore, S. Plate, R. Thomas, P. Wanderer, and K. C. Wu, in Proceedings of the Particle Accelerator Conference, Portland, OR, 2003 (IEEE, Piscataway, NJ, 2003).

[24] V. Balbekov et al., http://www-mucool.fnal.gov/mcnotes/ public/pdf/muc0202/muc0202.pdf

[25] A. Artamonov, Ya. Derbenev, and N. Inozemtsev, Sov. Phys. Tech. Phys. 34, 380 (1989).

[26] A. Artamonov, Ya. Derbenev, and N. Inozemtsev, Sov. Phys. Tech. Phys. 34, 495 (1990).

[27] L.D. Landau and E.M. Lifshits, Electrodynamics of Continuous Media (Pergamon, Oxford, 1984), 2nd ed.

[28] A. A. Kolomensky, Sov. At. Energy 19, 1511 (1965).

[29] Y. M. Ado and V. Balbekov, Sov. At. Energy 39, 40 (1971). 
[30] Ya. Derbenev and A. Skrinsky, Sov. Phys. Rev. 1, 165 (1981).

[31] http://en.wikipedia.org/wiki/Langevin_equation

[32] R. A. Alvarez, Rev. Sci. Instrum. 57, 2481 (1986).

[33] Z. D. Farkas et al., in Proceedings of the IX International Conference on High Energy Accelerators, Stanford, CA (SLAC Report No. SLAC-PUB-1453, 1974), p. 576.

[34] Rolland Johnson and Yaroslav Derbenev, DOE SBIR proposal No. 72180B03-I, 2003.
[35] Yaroslav Derbenev and Rolland P. Johnson, in Proceedings of the International Conference on Beam Cooling (COOL2003), Mt. Fuji, Japan, 2003, http:// members.aol.com/muonsinc/COOL03_6-d_rev1.pdf; Nucl. Instrum. Methods Phys. Res., Sect. A (to be published).

[36] L.D. Landau and E. M. Lifshits, Mechanics, Course of Theoretical Physics Vol. I (Pergamon, Oxford, 1978), 3rd ed. 